\title{
Cardiovascular Risk in Reproductive-aged Women With Polycystic Ovary Syndrome According to Body Mass Index: a Systematic Review and Meta-analysis
}

\section{Chenchen Zhuang}

Lanzhou University Second Hospital https://orcid.org/0000-0003-1746-6731

\section{Qiongying Wang}

Lanzhou University Second Hospital

\section{Wenjuan Wang}

Lanzhou University Second Hospital

Runmin Sun

Lanzhou University Second Hospital

\section{Xiaofang Zhang}

Lanzhou University Second Hospital

Jing Yu ( $\nabla$ ery_jyu@lzu.edu.cn )

Lanzhou University Second Hospital

\section{Research}

Keywords: PCOS, cardiovascular risk, reproductive-age, dyslipidemia, blood pressure

Posted Date: February 23rd, 2021

DOI: https://doi.org/10.21203/rs.3.rs-230193/v1

License: (c) (i) This work is licensed under a Creative Commons Attribution 4.0 International License.

Read Full License 


\section{Abstract}

Background: To evaluate the association between cardiovascular risk and reproductive-aged women with polycystic ovary syndrome (PCOS) according to body mass index (BMI) categories.

Methods: A literature search was conducted in MEDLINE, EMBASE, Cochrane Library and PubMed. Blood pressure (BP) [including systolic BP (SBP), diastolic BP (DBP) and hypertension prevalence] and plasma lipids [triglyceride, high-density lipoprotein (HDL)-cholesterol, nonHDL-cholesterol and low-density lipoprotein (LDL)-cholesterol] were calculated at different BMI levels.

Results: SBP [MD (95\% Cl): $2.96 \mathrm{mmHg}(1.62,4.30), \mathrm{P}<0.001]$ and DBP [MD $(95 \% \mathrm{Cl}): 1.64 \mathrm{mmHg}(0.03$, 3.24), $P=0.046]$ increased in reproductive-aged women with PCOS, however, hypertension prevalence had no difference [OR $(95 \% \mathrm{Cl}): 1.56(0.76,3.06), \mathrm{P}=0.198]$. HDL-cholesterol were [SMD $(95 \% \mathrm{Cl}):-0.24$ $(-0.39,-0.09), P=0.002]$ lower and triglyceride [SMD $(95 \% \mathrm{Cl}): 0.37(0.26,0.48), P<0.001]$ higher in reproductive PCOS women. Besides, LDL-cholesterol [SMD $(95 \% \mathrm{Cl}): 0.25(0.17,0.34), \mathrm{P}<0.001]$ and nonHDL-cholesterol [SMD (95\% Cl): $0.38(0.29,0.47), \mathrm{P}<0.001]$ were higher in reproductive-aged PCOS. In the subgroup analysis, SBP and LDL-cholesterol were increased in BMI of $<25 \mathrm{~kg} / \mathrm{m}^{2}$ and $\mathrm{BMI}$ of $\geq 30$ $\mathrm{kg} / \mathrm{m}^{2}$, but had no difference in BMl of $25-<30 \mathrm{~kg} / \mathrm{m}^{2}$. Triglyceride and nonHDL-cholesterol increased in all BMI categories. However, DBP and hypertension prevalence had no difference in all BMI categories.

Conclusion: High baseline BP and dyslipidemia is represented in reproductive PCOS. Besides, reproductive PCOS women are significantly associated with increased cardiovascular risk independent of BMl categories.

\section{Background}

Polycystic ovary syndrome (PCOS) is one of the most common female endocrinopathies, and is a highly prevalent disorder affecting about $7-14 \%$ of reproductive women $[1,2]$. The clinical manifestations of PCOS are heterogeneous, however, the hallmarks of the syndrome remain anovulation, insulin resistance and androgen excess. Moreover, each of these features is responsible for the promotion of cardiovascular risk in this population.

The American Society for Reproductive Medicine Practice Committee reported cardiovascular risk was increased in women with PCOS [3]. The current studies showed that cardiovascular risk factors were more frequent among women with PCOS compared to non-PCOS women $[4,5]$. This set of major risk factors includes age, sex, body mass index (BMI) category, low-density lipoprotein cholesterol (LDL-C) or triglyceride (TG), low high-density lipoprotein cholesterol (HDL-C), high systolic blood pressure (SBP) or diastolic blood pressure (DBP), diabetes mellitus, tobacco smoking and family history of early cardiovascular disease (CVD). Apart from the deleterious effects of PCOS per se, individual risk markers including hypertension and lipid profile are important mediators of the further cardiovascular outcomes. Blood pressure and lipid levels are nearly always included in risk assessment algorithms because of their established independent contributions to cardiovascular risk prediction [6]. The studies of early 
cardiovascular epidemiology focused on risk factors related to atherosclerosis, which begins at a young age. Detection of traditional cardiovascular risk factors and alternative markers of atherosclerosis in women with PCOS at a young age increase the risk for symptomatic cardiovascular risk $[7,8]$. Therefore, PCOS may represent an important key to lipid alternations starting during adolescence or fertile age.

Dyslipidemia is common in young adult women with PCOS, and the disorder may be the most common cause of dyslipidemia in women before the age of 40 years $[9,10]$. Moreover, atherosclerotic appears early life during life, this confirms the need to assess and eventually treat altered lipid profile in young women with PCOS [7]. LDL-C is considered to be the primary target to reduce cardiovascular risk, prevalence metabolic syndrome in women with PCOS has concentrated attention on TG and HDL-C, with relatively little attention to other lipid changes $[11,12]$. In addition, Rotterdam guidelines suggested evaluation for metabolic syndrome and indirectly indicated the need to measure only HDL-C and TG with relatively little attention to other lipid parameters [13]. The Androgen Excess and PCOS Society guidelines have reported that different LDL-C cutoff values depends on the degree of cardiovascular risk of women with PCOS [7]. However, many clinicians do not measure lipid levels in PCOS or continue measuring only HDL-C and TG.

The effect of obesity on PCOS and PCOS on obesity is complex. Although it is agreed that the prevalence of increased BMI is high in PCOS $[14,15]$. A previous meta-analysis showed that obesity was more prevalent in women with PCOS than in women without PCOS [16]. PCOS occurs both in obese and lean women. However, studies of cardiovascular risk in PCOS have not distinguished effects of BMI categories from those of PCOS. There is little consensus that the possible increased cardiovascular risk, is merely related to obesity. In the fact, lipid profile may differ in their association with lipid profile and PCOS potentially. Based on BMI categories, our meta-analysis classified as normal weight $\left(18.5-<25 \mathrm{~kg} / \mathrm{m}^{2}\right)$, overweight $\left(25<30 \mathrm{~kg} / \mathrm{m}^{2}\right)$, and obesity $\left(\geq 30 \mathrm{~kg} / \mathrm{m}^{2}\right)$ [17]. However, there is no met studies to report underweight $\left(<18.5 \mathrm{~kg} / \mathrm{m}^{2}\right)$ women with PCOS. These standard categories have been increasingly used in published studies of BMI levels and PCOS, but the literature reporting these results has not been systematically reviewed.

Therefore, the present meta-analysis of surrogate markers (i.e. blood pressure, lipids and hypertension prevalence) regarding cardiovascular risk factors shows the effect of different BMI levels in reproductiveaged women with PCOS. To reduce biases that may be present in all meta-analyses, only studies with BMI matching and having sufficient numbers of subjects were included. To our knowledge, this is the first meta-analysis to investigate the association between reproductive-aged women with PCOS and cardiovascular risk factors according to BMI levels.

\section{Methods}

The present study was approved by the Ethics Committee Board of Lanzhou University Second Hospital (D2019-098) and conducted in accordance with the Preferred Reporting Items for Preferred Reporting Items for Systematic Reviews and Meta-Analyses (PRISMA) [18]. 


\section{Search strategy}

We searched Cochrane Library, PubMed, EMBASE and MEDLINE databases to 9 November 2020 for full text articles in English describing trials and prospective cohort studies in reproductive-aged women that assessed the association between cardiovascular risk factors with PCOS according to BMI categories. The search terms were used as follows: (polycystic ovary syndrome or PCOS) and (cardiovascular risk). The search strategy was limited to English language papers.

\section{Study selection}

We used a mix of medical subject heading (MeSH) and keywords including polycystic ovary syndrome, Stein Leventhal, cardiovascular risk, blood pressure, hypertension, lipoprotein, dyslipidemia, hyperlipidemia. A manual search of the list of references of all identified studies and review articles was performed for additional relevant studies.

The selection criteria of the retrieved articles in our meta-analysis were as follows: 1) case-control studies or cohort studies; 2) reproductive-aged women with PCOS by the National Institutes of Health or Rotterdam criteria; 4) no comorbidities; 5) no evidence of an androgen-secreting tumor, congenital adrenal hyperplasia, or medication altering androgen metabolism or lipids; 6) each article with BMI matching. Each study had to have evaluated $\geq 30$ adults PCOS women $\geq 18$ years but $\leq 45$ years (to avoid the perimenopause transition). Control subjects of the same catchment area, and found to be without PCOS.

The exclusion criteria of the meta-analysis were as follows: 1) case-only studies; 2) studies with incomplete data; 3 ) meta-analysis, letters, reviews, and editorial articles. Nonpatient-examined community control studies were excluded, recognizing that PCOS occurs infrequently and that some phenotypic elements of PCOS can occur in regularly menstruating women.

\section{Assessment of study quality}

Included studies were assessed for quality using the Newcastle-Ottawa Scale (NOS), a validated scale for meta-analysis of observational studies [19]. We scored (max, nine points) the following items important for risk of bias assessment in non-randomized cohort studies: representativeness of the exposed cohort; adequate selection of controls; adequate definition of the outcome; adequacy of follow-up; comparability of exposed and non-exposed women (two points).

\section{Data extraction}

Authors of studies eligible for inclusion in present meta-analysis were invited to join our study and share their data. When not reported in the individual study, nonHDL-C was calculated as total cholesterol minus HDL-C. Data extraction was independently performed by three investigators (C.C.Z., Q.Y.W., W.J.W). Disagreement was resolved by consensus. 


\section{Statistical analysis}

The dichotomous outcome measure was the proportion of women with hypertension prevalence. Data were presented as odds ratio (OR, $95 \%$ confidence interval $[\mathrm{CI}])$. Continuous normally distributed data were summarized with a mean and standard deviation (SD), and if non-normally distributes a median and interquartile range (IQR) were used. If the outcome was measured on the same scale, we used weighted mean difference (MD) and $95 \% \mathrm{Cl}$. Otherwise, standardized mean difference (SMD) and $95 \% \mathrm{Cl}$ were calculated. Heterogeneity between the studies was assessed using $R$ tests $(R \otimes 50 \%$ was considered substantial heterogeneity). When the $R$ statistic was lower than $30 \%$ and $P<0.10$, a fixed-effect model was used; otherwise, a random effects model was used. We assessed publication bias through Egger's regression test. Analyses were performed by STATA statistical software version 14.0 (StataCorp, College Station, TX, USA). $p$-Value $<0.05$ were considered statistically significant for all analyses except heterogeneity tests.

\section{Results}

\section{Study design and analysis characteristics}

The search yielded 593 potential reports as shown in Fig. 1. After removal of duplicates, 556 records remained. Initial screening of the title and abstract resulted in the exclusion of 400 references and 156 studies proceeded to detailed evaluation. After further examination, 34 studies met the inclusion criteria and were included in the meta-analysis [8, 20-52].

The basic characteristics of each study were summarized in Table 1. A total of 34 studies including 12711 subjects were included in the present meta-analysis. The present meta-analysis was assessed blood pressure, prevalence of hypertension, TG, HDL-C, nonHDL-C and LDL-C according to BMI categories. 
Table 1

Characteristics of included studies in the meta-analysis

\begin{tabular}{|c|c|c|c|c|c|c|}
\hline Author/year & Country & Design & $\begin{array}{l}\text { Participants } \\
\text { age (years) }\end{array}$ & $\begin{array}{l}\text { PCOS } \\
\text { vs. } \\
\text { control } \\
\text { (n) }\end{array}$ & $\begin{array}{l}\text { BMI } \\
\text { level } \\
\left(\mathrm{kg} / \mathrm{m}^{2}\right)\end{array}$ & Outcomes \\
\hline \multirow[t]{2}{*}{ Adali (2010) } & Turkey & PC & $\begin{array}{l}\text { PCOS: } \\
24.73 \pm \\
2.91\end{array}$ & $\begin{array}{l}26 \text { vs. } \\
25\end{array}$ & $\begin{array}{l}\text { PCOS: } \\
24.4 \pm \\
4.23\end{array}$ & $\begin{array}{l}\text { BP, HDL-C, LDL-C, } \\
\text { TG }\end{array}$ \\
\hline & & & $\begin{array}{l}\text { Control: } \\
13.04 \pm \\
1.11\end{array}$ & & $\begin{array}{l}\text { Control: } \\
23.9 \pm \\
3.95\end{array}$ & \\
\hline \multirow[t]{2}{*}{ Akram (2010) } & Pakistan & $\mathrm{RC}$ & $\begin{array}{l}\text { PCOS: } 20- \\
39\end{array}$ & $\begin{array}{l}50 \text { vs. } \\
30\end{array}$ & $\begin{array}{l}\text { PCOS: } \\
23.6 \pm \\
0.50\end{array}$ & HDL-C, LDL-C, TG \\
\hline & & & $\begin{array}{l}\text { Control: } \\
20-39\end{array}$ & & $\begin{array}{l}\text { Control: } \\
23.5 \pm \\
0.71\end{array}$ & \\
\hline \multirow[t]{2}{*}{$\begin{array}{l}\text { Alexandraki } \\
(2006)\end{array}$} & Greece & PC & $\begin{array}{l}\text { PCOS: } \\
25.41 \pm \\
0.80\end{array}$ & $\begin{array}{l}27 \text { vs. } \\
27\end{array}$ & $\begin{array}{l}\text { PCOS: } \\
27.42 \pm \\
1.12\end{array}$ & $\begin{array}{l}\text { BP, HDL-C, LDL-C, } \\
\text { TG }\end{array}$ \\
\hline & & & $\begin{array}{l}\text { Control: } \\
27.33 \pm \\
0.83\end{array}$ & & $\begin{array}{l}\text { Control: } \\
25.05 \pm \\
1.19\end{array}$ & \\
\hline \multirow[t]{2}{*}{ Arikan (2007) } & Turkey & PC & $\begin{array}{l}\text { PCOS: } \\
22.82 \pm \\
5.53\end{array}$ & $\begin{array}{l}39 \text { vs. } \\
30\end{array}$ & $\begin{array}{l}\text { PCOS: } \\
21.48 \pm \\
6.50\end{array}$ & HDL-C, LDL-C, TG \\
\hline & & & $\begin{array}{l}\text { Control: } \\
24.64 \pm \\
4.22\end{array}$ & & $\begin{array}{l}\text { Control: } \\
20.90 \pm \\
6.04\end{array}$ & \\
\hline \multirow[t]{2}{*}{ Berneis (2006) } & Italy & PC & $\begin{array}{l}\text { PCOS: } 25.1 \\
\pm 4.2\end{array}$ & $\begin{array}{l}30 \text { vs. } \\
24\end{array}$ & $\begin{array}{l}\text { PCOS: } \\
28.4 \pm \\
5.8\end{array}$ & HDL-C, LDL-C, TG \\
\hline & & & $\begin{array}{l}\text { Control: } \\
25.5 \pm 3\end{array}$ & & $\begin{array}{l}\text { Control: } \\
28 \pm 4.4\end{array}$ & \\
\hline \multirow[t]{2}{*}{$\begin{array}{l}\text { Cascella } \\
(2006)\end{array}$} & Italy & PC & $\begin{array}{l}\text { PCOS: } 21.9 \\
\pm 2.7\end{array}$ & $\begin{array}{l}50 \text { vs. } \\
50\end{array}$ & $\begin{array}{l}\text { PCOS: } \\
24.6 \pm \\
2.5\end{array}$ & $\begin{array}{l}\text { BP, HDL-C, LDL-C, } \\
\text { TG }\end{array}$ \\
\hline & & & $\begin{array}{l}\text { Control: } \\
22.2 \pm 2.8\end{array}$ & & $\begin{array}{l}\text { Control: } \\
24.4 \pm \\
2.8\end{array}$ & \\
\hline
\end{tabular}

PCOS, polycystic ovary syndrome; BMI, body mass index; PC, prospectively cohort study; RC, retrospectively cohort study, BP, blood pressure (including systolic and diastolic blood pressure); SBP, systolic blood pressure; HDL-C, high-density lipoprotein cholesterol; LDL-C, low-density lipoprotein cholesterol; TG, triglycerides. 


\begin{tabular}{|c|c|c|c|c|c|c|}
\hline Author/year & Country & Design & $\begin{array}{l}\text { Participants } \\
\text { age (years) }\end{array}$ & $\begin{array}{l}\text { PCOS } \\
\text { vs. } \\
\text { control } \\
\text { (n) }\end{array}$ & $\begin{array}{l}\text { BMI } \\
\text { level } \\
\left(\mathrm{kg} / \mathrm{m}^{2}\right)\end{array}$ & Outcomes \\
\hline \multirow[t]{2}{*}{$\begin{array}{l}\text { Cetinakalp } \\
(2009)\end{array}$} & Turkey & PC & $\begin{array}{l}\text { PCOS: } \\
24.58 \pm \\
4.61\end{array}$ & $\begin{array}{l}129 \text { vs. } \\
91\end{array}$ & $\begin{array}{l}\text { PCOS: } \\
24.47 \pm \\
4.64\end{array}$ & HDL-C, LDL-C, TG \\
\hline & & & $\begin{array}{l}\text { Control: } \\
25.48 \pm \\
3.38\end{array}$ & & $\begin{array}{l}\text { Control: } \\
24.2 \pm \\
3.31\end{array}$ & \\
\hline \multirow[t]{2}{*}{$\begin{array}{l}\text { Cussons } \\
(2009)\end{array}$} & Australia & PC & $\begin{array}{l}\text { PCOS: } 30.4 \\
\pm 5.54\end{array}$ & $\begin{array}{l}19 \text { vs. } \\
19\end{array}$ & $\begin{array}{l}\text { PCOS: } \\
24.1 \pm \\
2.9\end{array}$ & $\begin{array}{l}\text { BP, HDL-C, LDL-C } \\
\text { TG }\end{array}$ \\
\hline & & & $\begin{array}{l}\text { Control: } \\
34.44 \pm 7.8\end{array}$ & & $\begin{array}{l}\text { Control: } \\
22.9 \pm \\
3.2\end{array}$ & \\
\hline \multirow[t]{2}{*}{$\begin{array}{l}\text { Diamanti- } \\
\text { Kandarakis } \\
(2006)\end{array}$} & Greece & PC & $\begin{array}{l}\text { PCOS: } \\
25.64 \pm \\
0.86\end{array}$ & $\begin{array}{l}25 \text { vs. } \\
25\end{array}$ & $\begin{array}{l}\text { PCOS: } \\
29.08 \pm \\
1.43\end{array}$ & BP, HDL-C \\
\hline & & & $\begin{array}{l}\text { Control: } \\
27.52 \pm \\
1.02\end{array}$ & & $\begin{array}{l}\text { Control: } \\
26.22 \pm \\
1.16\end{array}$ & \\
\hline \multirow[t]{2}{*}{$\begin{array}{l}\text { El-Kannishy } \\
(2009)\end{array}$} & Egypt & PC & $\begin{array}{l}\text { PCOS: } 25.2 \\
\pm 3.6\end{array}$ & $\begin{array}{l}14 \text { vs. } \\
10\end{array}$ & $\begin{array}{l}\text { PCOS: } \\
22.8 \pm \\
2.1\end{array}$ & HDL-C, LDL-C, TG \\
\hline & & & $\begin{array}{l}\text { Control: } \\
24.4 \pm 4.07\end{array}$ & & $\begin{array}{l}\text { Control: } \\
21.9 \pm \\
2.97\end{array}$ & \\
\hline \multirow[t]{2}{*}{$\begin{array}{l}\text { Erdogan } \\
(2007)\end{array}$} & Turkey & PC & $\begin{array}{l}\text { PCOS: } \\
24.27 \pm \\
5.44\end{array}$ & $\begin{array}{l}68 \text { vs. } \\
26\end{array}$ & $\begin{array}{l}\text { PCOS: } \\
24.41 \pm \\
5.43\end{array}$ & HDL-C, LDL-C, TG \\
\hline & & & $\begin{array}{l}\text { Control: } \\
26.41 \pm \\
5.65\end{array}$ & & $\begin{array}{l}\text { Control: } \\
23.35 \pm \\
5.04\end{array}$ & \\
\hline \multirow[t]{2}{*}{$\begin{array}{l}\text { Erdogan } \\
(2009)\end{array}$} & Turkey & PC & $\begin{array}{l}\text { PCOS: } \\
24.07 \pm \\
1.32\end{array}$ & $\begin{array}{l}88 \text { vs. } \\
119\end{array}$ & $\begin{array}{l}\text { PCOS: } \\
24.38 \pm \\
4.13\end{array}$ & HDL-C, LDL-C, TG \\
\hline & & & $\begin{array}{l}\text { Control: } \\
25.01 \pm \\
2.05\end{array}$ & & $\begin{array}{l}\text { Control: } \\
23.47 \pm \\
4.12\end{array}$ & \\
\hline
\end{tabular}

PCOS, polycystic ovary syndrome; BMI, body mass index; PC, prospectively cohort study; RC, retrospectively cohort study, BP, blood pressure (including systolic and diastolic blood pressure); SBP, systolic blood pressure; HDL-C, high-density lipoprotein cholesterol; LDL-C, low-density lipoprotein cholesterol; TG, triglycerides. 


\begin{tabular}{|c|c|c|c|c|c|c|}
\hline Author/year & Country & Design & $\begin{array}{l}\text { Participants } \\
\text { age (years) }\end{array}$ & $\begin{array}{l}\text { PCOS } \\
\text { vs. } \\
\text { control } \\
\text { (n) }\end{array}$ & $\begin{array}{l}\text { BMI } \\
\text { level } \\
\left(\mathrm{kg} / \mathrm{m}^{2}\right)\end{array}$ & Outcomes \\
\hline Joham (2014) & Australia & $\mathrm{RC}$ & $28-33$ & $\begin{array}{l}183 \text { vs. } \\
4638\end{array}$ & $<25$ & $\begin{array}{l}\text { Prevalence of } \\
\text { hypertension }\end{array}$ \\
\hline Joham (2014) & Australia & $\mathrm{RC}$ & $28-33$ & $\begin{array}{l}117 \text { vs. } \\
1742\end{array}$ & $\begin{array}{l}25.0- \\
29.9\end{array}$ & $\begin{array}{l}\text { Prevalence of } \\
\text { hypertension }\end{array}$ \\
\hline Joham (2014) & Australia & $\mathrm{RC}$ & $28-33$ & $\begin{array}{l}149 \text { vs. } \\
1181\end{array}$ & $\geq 30$ & $\begin{array}{l}\text { Prevalence of } \\
\text { hypertension }\end{array}$ \\
\hline Kargili (2010) & Turkey & $\mathrm{RC}$ & $\begin{array}{l}\text { PCOS: } 25.7 \\
\pm 5.5 \\
\text { Control: } \\
26.1 \pm 5.4\end{array}$ & $\begin{array}{l}168 \text { vs. } \\
52\end{array}$ & $\begin{array}{l}\text { PCOS: } \\
26.8 \pm \\
3.4 \\
\text { Control: } \\
25.4 \pm \\
2.8\end{array}$ & HDL-C, LDL-C, TG \\
\hline Ketel (2010) & Netherland & $\mathrm{RC}$ & $\begin{array}{l}\text { PCOS: } 28.6 \\
\pm 4.5 \\
\text { Control: } \\
27.7 \pm 5.3\end{array}$ & $\begin{array}{l}22 \\
\text { vs.14 }\end{array}$ & $\begin{array}{l}\text { PCOS: } \\
22.0 \pm \\
2.2 \\
\text { Control: } \\
22.2 \pm \\
1.7\end{array}$ & HDL-C, LDL-C, TG \\
\hline Ketel (2010) & Netherland & $\mathrm{RC}$ & $\begin{array}{l}\text { PCOS: } 30.3 \\
\pm 4.2 \\
\text { Control: } \\
28.6 \pm 5.3\end{array}$ & $\begin{array}{l}18 \text { vs. } \\
13\end{array}$ & $\begin{array}{l}\text { PCOS: } \\
36.2 \pm \\
5.9 \\
\text { Control: } \\
40.5 \pm \\
7.0\end{array}$ & HDL-C, LDL-C, TG \\
\hline Legro (2001) & USA & PC & $\begin{array}{l}\text { PCOS: } 25 \pm \\
6 \\
\text { Control: } 29 \\
\pm 7\end{array}$ & $\begin{array}{l}42 \text { vs. } \\
27\end{array}$ & $\begin{array}{l}\text { PCOS: } \\
23.1 \pm \\
2.4 \\
\text { Control: } \\
23.0 \pm \\
1.8\end{array}$ & $\begin{array}{l}\text { BP, HDL-C, LDL-C, } \\
\text { TG }\end{array}$ \\
\hline Legro (2001) & USA & PC & $\begin{array}{l}\text { PCOS: } 28 \pm \\
5 \\
\text { Control: } 32 \\
\pm 7\end{array}$ & $\begin{array}{l}153 \text { vs. } \\
35\end{array}$ & $\begin{array}{l}\text { PCOS: } \\
37.0 \pm \\
6.9 \\
\text { Control: } \\
37.7 \pm \\
6.4\end{array}$ & $\begin{array}{l}\text { BP, HDL-C, LDL-C, } \\
\text { TG }\end{array}$ \\
\hline
\end{tabular}

PCOS, polycystic ovary syndrome; BMI, body mass index; PC, prospectively cohort study; RC, retrospectively cohort study, BP, blood pressure (including systolic and diastolic blood pressure); SBP, systolic blood pressure; HDL-C, high-density lipoprotein cholesterol; LDL-C, low-density lipoprotein cholesterol; TG, triglycerides. 


\begin{tabular}{|c|c|c|c|c|c|c|}
\hline Author/year & Country & Design & $\begin{array}{l}\text { Participants } \\
\text { age (years) }\end{array}$ & $\begin{array}{l}\text { PCOS } \\
\text { vs. } \\
\text { control } \\
\text { (n) }\end{array}$ & $\begin{array}{l}\text { BMl } \\
\text { level } \\
\left(\mathrm{kg} / \mathrm{m}^{2}\right)\end{array}$ & Outcomes \\
\hline Liang (2012) & Taiwan & PC & $\begin{array}{l}\text { PCOS: } 27 \pm \\
6.4 \\
\text { Control: } \\
29.0 \pm 5.1\end{array}$ & $\begin{array}{l}110 \text { vs. } \\
20\end{array}$ & $\begin{array}{l}\text { PCOS: } \\
31.1 \pm \\
3.9 \\
\text { Control: } \\
30.4 \pm \\
3.7\end{array}$ & $\begin{array}{l}\text { Prevalence of } \\
\text { hypertension, HDL- } \\
\text { C, LDL-C, TG }\end{array}$ \\
\hline Liang (2012) & Taiwan & PC & $\begin{array}{l}\text { PCOS: } 26.8 \\
\pm 5.1 \\
\text { Control: } \\
28.1 \pm 4.2\end{array}$ & $\begin{array}{l}110 \text { vs. } \\
50\end{array}$ & $\begin{array}{l}\text { PCOS: } \\
20.6 \pm \\
2.0 \\
\text { Control: } \\
20.4 \pm \\
2.0\end{array}$ & $\begin{array}{l}\text { Prevalence of } \\
\text { hypertension, HDL- } \\
\text { C, LDL-C, TG }\end{array}$ \\
\hline $\begin{array}{l}\text { Luque- } \\
\text { Ramirez } \\
\text { (2007) }\end{array}$ & Spain & PC & $\begin{array}{l}\text { PCOS: } 23.0 \\
\pm 5.4 \\
\text { Control: } \\
24.8 \pm 6.0\end{array}$ & 11 vs. 8 & $\begin{array}{l}\text { PCOS: } \\
22.2 \pm \\
2.0 \\
\text { Control: } \\
21.3 \pm \\
1.3\end{array}$ & HDL-C, LDL-C, TG \\
\hline $\begin{array}{l}\text { Luque- } \\
\text { Ramirez } \\
\text { (2007) }\end{array}$ & Spain & PC & $\begin{array}{l}\text { PCOS: } 23.6 \\
\pm 4.6 \\
\text { Control: } \\
29.3 \pm 10.3\end{array}$ & 13 vs. 4 & $\begin{array}{l}\text { PCOS: } \\
27.5 \pm \\
1.8 \\
\text { Control: } \\
27.4 \pm \\
1.5\end{array}$ & HDL-C, LDL-C, TG \\
\hline $\begin{array}{l}\text { Luque- } \\
\text { Ramirez } \\
\text { (2007) }\end{array}$ & Spain & PC & $\begin{array}{l}\text { PCOS: } 26.3 \\
\pm 6.7 \\
\text { Control: } \\
28.5 \pm 5.8\end{array}$ & 16 vs. 8 & $\begin{array}{l}\text { PCOS: } \\
35.8 \pm \\
3.9 \\
\text { Control: } \\
35.5 \pm \\
3.2\end{array}$ & HDL-C, LDL-C, TG \\
\hline Macut (2008) & Serbia & PC & $\begin{array}{l}\text { PCOS: } 23.1 \\
\pm 5.1 \\
\text { Control: } \\
24.6 \pm 4.1\end{array}$ & $\begin{array}{l}75 \text { vs. } \\
51\end{array}$ & $\begin{array}{l}\text { PCOS: } \\
24.9 \pm \\
4.7 \\
\text { Control: } \\
23.7 \pm \\
4.0\end{array}$ & HDL-C, LDL-C, TG \\
\hline
\end{tabular}

PCOS, polycystic ovary syndrome; BMI, body mass index; PC, prospectively cohort study; RC, retrospectively cohort study, BP, blood pressure (including systolic and diastolic blood pressure); SBP, systolic blood pressure; HDL-C, high-density lipoprotein cholesterol; LDL-C, low-density lipoprotein cholesterol; TG, triglycerides. 


\begin{tabular}{|c|c|c|c|c|c|c|}
\hline Author/year & Country & Design & $\begin{array}{l}\text { Participants } \\
\text { age (years) }\end{array}$ & $\begin{array}{l}\text { PCOS } \\
\text { vs. } \\
\text { control } \\
\text { (n) }\end{array}$ & $\begin{array}{l}\text { BMI } \\
\text { level } \\
\left(\mathrm{kg} / \mathrm{m}^{2}\right)\end{array}$ & Outcomes \\
\hline Moran (2009) & Australia & PC & $\begin{array}{l}\text { PCOS: } 34.1 \\
\pm 6.9 \\
\text { Control: } \\
33.8 \pm 6.8\end{array}$ & $\begin{array}{l}80 \text { vs. } \\
27\end{array}$ & $\begin{array}{l}\text { PCOS: } \\
36.0 \pm \\
6.6 \\
\text { Control: } \\
37.4 \pm \\
5.6\end{array}$ & HDL-C, LDL-C, TG \\
\hline Mayer (2005) & Australia & PC & $\begin{array}{l}\text { PCOS: } 32.7 \\
\pm 1.8 \\
\text { Control: } \\
33.2 \pm 2.3\end{array}$ & $\begin{array}{l}100 \text { vs. } \\
20\end{array}$ & $\begin{array}{l}\text { PCOS: } \\
37.3 \pm \\
2.43 \\
\text { Control: } \\
36.7 \pm \\
1.28\end{array}$ & HDL-C, LDL-C, TG \\
\hline $\mathrm{Ni}(2009)$ & China & PC & $\begin{array}{l}\text { PCOS: } 27 \\
\text { Control: } 28\end{array}$ & $\begin{array}{l}578 \text { vs. } \\
281\end{array}$ & $\begin{array}{l}\text { PCOS: } \\
21.9 \\
\text { Control: } \\
21.9\end{array}$ & $\mathrm{BP}, \mathrm{TG}$ \\
\hline Oral (2008) & Turkey & PC & $\begin{array}{l}\text { PCOS: } 23.9 \\
\pm 3.3 \\
\text { Control: } \\
24.2 \pm 3.9\end{array}$ & $\begin{array}{l}48 \text { vs. } \\
43\end{array}$ & $\begin{array}{l}\text { PCOS: } \\
24.1 \pm \\
2.9 \\
\text { Control: } \\
24.0 \pm \\
1.9\end{array}$ & HDL-C, LDL-C, TG \\
\hline Orio (2004) & Italy & PC & $\begin{array}{l}\text { PCOS: } 22.2 \\
\pm 2.5 \\
\text { Control: } \\
22.6 \pm 2.3\end{array}$ & $\begin{array}{l}30 \text { vs. } \\
30\end{array}$ & $\begin{array}{l}\text { PCOS: } \\
22.4 \pm \\
2.1 \\
\text { Control: } \\
22.1 \pm \\
1.8\end{array}$ & $\begin{array}{l}\text { BP, HDL-C, LDL-C, } \\
\text { TG }\end{array}$ \\
\hline $\begin{array}{l}\text { Philbois } \\
\text { (2018) }\end{array}$ & Brazil & PC & $\begin{array}{l}\text { PCOS: } 28.5 \\
\pm 5.2 \\
\text { Control: } \\
31.2 \pm 6.6\end{array}$ & $\begin{array}{l}30 \text { vs. } \\
30\end{array}$ & $\begin{array}{l}\text { PCOS: } \\
22.9 \pm \\
1.6 \\
\text { Control: } \\
23.5 \pm 3\end{array}$ & $\mathrm{BP}$ \\
\hline
\end{tabular}

PCOS, polycystic ovary syndrome; BMI, body mass index; PC, prospectively cohort study; RC, retrospectively cohort study, BP, blood pressure (including systolic and diastolic blood pressure); SBP, systolic blood pressure; HDL-C, high-density lipoprotein cholesterol; LDL-C, low-density lipoprotein cholesterol; TG, triglycerides. 


\begin{tabular}{|c|c|c|c|c|c|c|}
\hline Author/year & Country & Design & $\begin{array}{l}\text { Participants } \\
\text { age (years) }\end{array}$ & $\begin{array}{l}\text { PCOS } \\
\text { vs. } \\
\text { control } \\
\text { (n) }\end{array}$ & $\begin{array}{l}\text { BMI } \\
\text { level } \\
\left(\mathrm{kg} / \mathrm{m}^{2}\right)\end{array}$ & Outcomes \\
\hline \multirow[t]{2}{*}{ Rizzo (2011) } & \multirow[t]{2}{*}{ Italy } & \multirow[t]{2}{*}{ PC } & $\begin{array}{l}\text { PCOS: } 24 \pm \\
5\end{array}$ & \multirow[t]{2}{*}{$\begin{array}{l}350 \text { vs. } \\
90\end{array}$} & $\begin{array}{l}\text { PCOS: } \\
27 \pm 7\end{array}$ & \multirow[t]{2}{*}{$\begin{array}{l}\text { HDL-C, LDL-C, TG, } \\
\text { nonHDL-C }\end{array}$} \\
\hline & & & $\begin{array}{l}\text { Control: } 24 \\
\pm 3\end{array}$ & & $\begin{array}{l}\text { Control: } \\
27 \pm 4\end{array}$ & \\
\hline \multirow[t]{2}{*}{ Sasaki (2011) } & \multirow[t]{2}{*}{ Japan } & \multirow[t]{2}{*}{ PC } & $\begin{array}{l}\text { PCOS: } 30.2 \\
\pm 3.9\end{array}$ & \multirow[t]{2}{*}{$\begin{array}{l}54 \text { vs. } \\
24\end{array}$} & $\begin{array}{l}\text { PCOS: } \\
24.3 \pm \\
5.7\end{array}$ & \multirow[t]{2}{*}{$\begin{array}{l}\text { BP, HDL-C, LDL-C, } \\
\text { TG }\end{array}$} \\
\hline & & & $\begin{array}{l}\text { Control: } \\
31.5 \pm 4.4\end{array}$ & & $\begin{array}{l}\text { Control: } \\
22.2 \pm \\
3.4\end{array}$ & \\
\hline \multirow[t]{2}{*}{ Shroff (2007) } & \multirow[t]{2}{*}{ USA } & \multirow[t]{2}{*}{ PC } & $\begin{array}{l}\text { PCOS: } 32 \pm \\
6.5\end{array}$ & \multirow[t]{2}{*}{$\begin{array}{l}24 \text { vs. } \\
24\end{array}$} & $\begin{array}{l}\text { PCOS: } \\
36 \pm 5.4\end{array}$ & \multirow[t]{2}{*}{$\begin{array}{l}\text { Prevalence of } \\
\text { hypertension, HDL- } \\
\text { C, LDL-C, TG }\end{array}$} \\
\hline & & & $\begin{array}{l}\text { Control: } 36 \\
\pm 7.2\end{array}$ & & $\begin{array}{l}\text { Control: } \\
35 \pm 3.3\end{array}$ & \\
\hline \multirow[t]{2}{*}{ Soares (2009) } & \multirow[t]{2}{*}{ Brazil } & \multirow[t]{2}{*}{$\mathrm{PC}$} & $\begin{array}{l}\text { PCOS: } 24.5 \\
\pm 3.8\end{array}$ & \multirow[t]{2}{*}{$\begin{array}{l}40 \mathrm{vs} . \\
50\end{array}$} & $\begin{array}{l}\text { PCOS: } \\
22.7 \pm \\
3.3\end{array}$ & \multirow[t]{2}{*}{$\begin{array}{l}\text { BP, HDL-C, LDL-C, } \\
\text { TG }\end{array}$} \\
\hline & & & $\begin{array}{l}\text { Control: } \\
24.5 \pm 5.1\end{array}$ & & $\begin{array}{l}\text { Control: } \\
23.1 \pm \\
3.2\end{array}$ & \\
\hline \multirow[t]{2}{*}{ Sterling (2015) } & \multirow[t]{2}{*}{ Canada } & \multirow[t]{2}{*}{$\mathrm{RC}$} & PCOS: 33 & \multirow[t]{2}{*}{$\begin{array}{l}71 \mathrm{vs} . \\
323\end{array}$} & $\begin{array}{l}\text { PCOS: } \\
24.6\end{array}$ & \multirow[t]{2}{*}{$\begin{array}{l}\text { Prevalence of } \\
\text { hypertension }\end{array}$} \\
\hline & & & Control: 35 & & $\begin{array}{l}\text { Control: } \\
23.6\end{array}$ & \\
\hline \multirow[t]{2}{*}{ Tarkun (2004) } & \multirow[t]{2}{*}{ Turkey } & \multirow[t]{2}{*}{ PC } & $\begin{array}{l}\text { PCOS: } \\
23.45 \pm 4.3\end{array}$ & \multirow[t]{2}{*}{$\begin{array}{l}37 \text { vs. } \\
25\end{array}$} & $\begin{array}{l}\text { PCOS: } \\
23.85 \pm \\
3.26\end{array}$ & \multirow[t]{2}{*}{ HDL-C, LDL-C, TG } \\
\hline & & & $\begin{array}{l}\text { Control: } \\
24.4 \pm 4.07\end{array}$ & & $\begin{array}{l}\text { Control: } \\
22.9 \pm \\
2.97\end{array}$ & \\
\hline
\end{tabular}

PCOS, polycystic ovary syndrome; BMI, body mass index; PC, prospectively cohort study; RC, retrospectively cohort study, BP, blood pressure (including systolic and diastolic blood pressure); SBP, systolic blood pressure; HDL-C, high-density lipoprotein cholesterol; LDL-C, low-density lipoprotein cholesterol; TG, triglycerides. 


\begin{tabular}{|c|c|c|c|c|c|c|}
\hline Author/year & Country & Design & $\begin{array}{l}\text { Participants } \\
\text { age (years) }\end{array}$ & $\begin{array}{l}\text { Pcos } \\
\text { vs. } \\
\text { control } \\
\text { (n) }\end{array}$ & $\begin{array}{l}\text { BMI } \\
\text { level } \\
\left(\mathrm{kg} / \mathrm{m}^{2}\right)\end{array}$ & Outcomes \\
\hline Tiras (1999) & Turkey & $\mathrm{PC}$ & $\begin{array}{l}\text { PCOS: } 24.5 \\
\pm 6.0 \\
\text { Control: } \\
23.6 \pm 3.9\end{array}$ & $\begin{array}{l}35 \mathrm{vs.} \\
35\end{array}$ & $\begin{array}{l}\text { PCOS: } \\
22.9 \pm \\
4.2 \\
\text { Control: } \\
22.0 \pm \\
1.8\end{array}$ & HDL-C, LDL-C, TG \\
\hline $\begin{array}{l}\text { Vryonidou } \\
\text { (2005) }\end{array}$ & Greece & $\mathrm{PC}$ & $\begin{array}{l}\text { PCOS: } 23.9 \\
\pm 5.4 \\
\text { Control: } \\
24.7 \pm 5.3\end{array}$ & $\begin{array}{l}75 \text { vs. } \\
55\end{array}$ & $\begin{array}{l}\text { PCOS: } \\
27.3 \pm \\
7.0 \\
\text { Control: } \\
26.3 \pm \\
7.7\end{array}$ & $\begin{array}{l}\text { SBP, HDL-C, LDL-C, } \\
\text { TG }\end{array}$ \\
\hline Yildiz (2002) & Turkey & PC & $\begin{array}{l}\text { PCOS: } 22.9 \\
\pm 4.4 \\
\text { Control: } \\
24.8 \pm 4.2\end{array}$ & $\begin{array}{l}59 \text { vs. } \\
23\end{array}$ & $\begin{array}{l}\text { PCOS: } \\
23.0 \pm \\
2.4 \\
\text { Control: } \\
22.1 \pm \\
2.2\end{array}$ & HDL-C, TG \\
\hline $\begin{array}{l}\text { PCOS, polycy } \\
\text { retrospectivel } \\
\text { systolic blooc } \\
\text { cholesterol; T }\end{array}$ & $\begin{array}{l}\text { vary syn } \\
\text { ort study } \\
\text { sure; HDI } \\
\text { lycerides }\end{array}$ & $\begin{array}{l}\text { BMI, } \\
\text { lood pI } \\
\text { gh-den }\end{array}$ & $\begin{array}{l}\text { y mass ind } \\
\text { ure (includi } \\
\text { lipoprotein }\end{array}$ & $\begin{array}{l}\text { PC, pro } \\
\text { systoli } \\
\text { olester }\end{array}$ & $\begin{array}{l}\text { tively co } \\
\text { d diastol } \\
\text { DL-C, lov }\end{array}$ & $\begin{array}{l}\text { rt study; RC, } \\
\text { lood pressure); SBP } \\
\text { ensity lipoprotein }\end{array}$ \\
\hline
\end{tabular}

\section{Risk of bias and quality assessment}

The 34 studies included for systematic review were then reviewed with the NOS tool (Table 2). Some studies did not report on allocation concealment. Binding was impossible. One study scored seven out of nine points and one study scored six points, indicating high-quality. Three studies scored four points or less. 
Table 2

Assessment of methodological quality (based on Newcastle-Ottawa Scale)

\begin{tabular}{|c|c|c|c|c|}
\hline & $\begin{array}{l}\text { Selection (max } 4 \\
\text { stars) }\end{array}$ & $\begin{array}{l}\text { Comparability (max } \\
2 \text { stars) }\end{array}$ & $\begin{array}{l}\text { Exposure (max } 3 \\
\text { stars) }\end{array}$ & $\begin{array}{l}\text { Overall } \\
\text { quality }\end{array}$ \\
\hline Adali (2010) & $\star \star \star *$ & ** & $\star *$ & Good \\
\hline Akram (2010) & ** & $\star *$ & $\star *$ & Fair \\
\hline Alexandraki (2006) & $\star * *$ & ** & $\star *$ & Good \\
\hline Arikan (2007) & $\star * *$ & $\star \star$ & $\star *$ & Good \\
\hline Berneis (2006) & $\star \star \star \star$ & $\star \star$ & $\star \star$ & Good \\
\hline Cascella (2006) & $\star \star \star \star *$ & $\star *$ & $\star *$ & Good \\
\hline Cetinakalp (2009) & $\star \star$ & $\star \star$ & $\star \star$ & Fair \\
\hline Cussons (2009) & $\star \star$ & $\star \star$ & * & Fair \\
\hline $\begin{array}{l}\text { Diamanti-Kandarakis } \\
(2006)\end{array}$ & $\star \star$ & $\star *$ & * & Fair \\
\hline El-Kannishy (2009) & 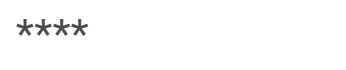 & ** & $\star \star$ & Good \\
\hline Erdogan (2007) & ** & * & $\star *$ & Fair \\
\hline Erdogan (2009) & 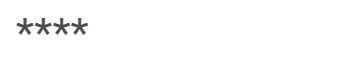 & ** & ** & Good \\
\hline Joham (2014) & $\star \star \star$ & ** & ** & Fair \\
\hline Kargili (2010) & ** & $\star \star *$ & $\star \star *$ & Fair \\
\hline Ketel (2010) & 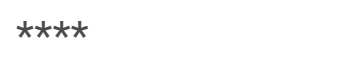 & $\star \star$ & $\star *$ & Good \\
\hline Legro (2001) & $\star * \star$ & * & $\star *$ & Good \\
\hline Liang (2012) & $\star * \star$ & * & $\star *$ & Good \\
\hline $\begin{array}{l}\text { Luque-Ramirez } \\
(2007)\end{array}$ & $\star \star \star *$ & ** & $\star \star$ & Good \\
\hline Macut (2008) & $\star \star$ & $\star *$ & $\star *$ & Fair \\
\hline Moran (2009) & $\star \star \star$ & $\star \star$ & $\star \star$ & Good \\
\hline Mayer (2005) & $\star \star \star \star$ & $\star \star$ & $\star \star$ & Good \\
\hline $\mathrm{Ni}(2009)$ & $\star \star$ & * & $\star *$ & Fair \\
\hline Oral (2008) & $\star \star$ & * & $\star *$ & Fair \\
\hline Orio (2004) & $\star \star \star *$ & $\star *$ & $\star *$ & Good \\
\hline Philbois (2018) & ** & ** & ** & Fair \\
\hline
\end{tabular}




\begin{tabular}{|c|c|c|c|c|}
\hline & $\begin{array}{l}\text { Selection (max } 4 \\
\text { stars) }\end{array}$ & $\begin{array}{l}\text { Comparability (max } \\
2 \text { stars) }\end{array}$ & $\begin{array}{l}\text { Exposure (max } 3 \\
\text { stars) }\end{array}$ & $\begin{array}{l}\text { Overall } \\
\text { quality }\end{array}$ \\
\hline Rizzo (2011) & ** & ** & ** & Fair \\
\hline Sasaki (2011) & $\star \star *$ & ** & $\star \star$ & Good \\
\hline Shroff (2007) & $\star \star *$ & ** & ** & Good \\
\hline Soares (2009) & ** & ** & ** & Fair \\
\hline Sterling (2015) & ** & * & ** & Fair \\
\hline Tarkun (2004) & ** & ** & ** & Fair \\
\hline Tiras (1999) & ** & ** & ** & Fair \\
\hline Vryonidou (2005) & $\star \star \star \star \star ~$ & ** & ** & Good \\
\hline Yildiz (2002) & $\star \star$ & * & ** & Fair \\
\hline
\end{tabular}

\section{SELECTION}

(1) Is the case definition adequate? (a) yes, with independent validation*, (b) yes, e.g. record linkage or based on self-reports, (c) no description

(2) Representativeness of the cases: (a) consecutive or obviously representative series of cases*, (b) potential for selection biases or not stated

(3) Selection of controls: (a) community controls*, (b) hospital controls, (c) no description

(4) Definition of controls: (a) no history of disease (end-point)*, (b) no description of source

\section{COMPARABILITY}

Comparability of cases and controls on basis of design or analysis: (a) study controls for _ (most important factor)*, (b) study controls for any additional factor* (could be modified to indicate specific control for a second factor)

\section{EXPOSURE}

(1) Ascertainment of exposure: (a) secure record (e.g. surgical record)*, (b) structured interview where blind to case/control status*, (c) interview not blinded to case/control status, (d) written self-report or medical record only, (e) no description.

(2) Same method of ascertainment for cases and controls: (a) yes*, (b) no.

(3) Non-response rate: (a) same rate for both groups*, (b) non-respondents describe, (c) rate different and no designation.

OVERALL QUALITY

Good quality: 3 or 4 stars in selection domain AND 1 or 2 stars in comparability domain AND 2 or 3 stars in exposure domain.

Fair quality: 2 stars in selection domain AND 1 or 2 stars in comparability domain AND 2 or 3 stars in exposure domain. 


\section{Selection (max $4 \quad$ Comparability (max stars)

Poor quality: 0 or 1 star in selection domain OR 0 star in comparability domain OR 0 or 1 star in exposure domain.

\section{Blood pressure}

Figures 2-4 are forest plots summarizing comparison of SBP, DBP and prevalence of hypertension, respectively. Because the aim was assessed the effect of BMI categories on cardiovascular risk in reproductive women with PCOS, we analyzed the outcomes based on BMI categories.

As shown in Figs. 2 and 3, SBP [MD (95\% Cl): 2.96 mmHg (1.62, 4.30), $\mathrm{P}<0.001]$ and DBP [MD (95\% Cl): $1.64 \mathrm{mmHg}(0.03,3.24), P=0.046]$ increased in reproductive-aged women with PCOS. In the subgroup analysis of SBP, BMI of $<25 \mathrm{~kg} / \mathrm{m}^{2}$ [MD $\left.(95 \% \mathrm{Cl}): 3.02 \mathrm{mmHg}(1.11,4.94), \mathrm{P}<0.002\right]$ and BMI of $\geq 30$ $\mathrm{kg} / \mathrm{m}^{2}$ [MD (95\% Cl): $\left.4.27 \mathrm{mmHg}(0.50,8.04), \mathrm{P}<0.026\right]$ showed significant results, while BMI of $25<30$ $\mathrm{kg} / \mathrm{m}^{2}[\mathrm{MD}(95 \% \mathrm{Cl}): 1.56 \mathrm{mmHg}(-0.60,3.71), \mathrm{P}=0.157]$ did not show positive result. However, in the subgroup analysis, DBP did not increase in $\mathrm{BMI}$ of $<25 \mathrm{~kg} / \mathrm{m}^{2}$ [MD $(95 \% \mathrm{Cl}): 1.75 \mathrm{mmHg}(-0.23,3.75), \mathrm{P}=$ 0.083 ], BMl of $\geq 30 \mathrm{~kg} / \mathrm{m}^{2}$ [MD (95\% Cl): $-0.24 \mathrm{mmHg}(-3.16,2.69), \mathrm{P}=0.873$ ], and $\mathrm{BMI}$ of $25-<30 \mathrm{~kg} / \mathrm{m}^{2}$ [MD $(95 \% \mathrm{Cl}): 3.13 \mathrm{mmHg}(-0.98,7.24), \mathrm{P}=0.136]$.

As shown in Fig. 4, prevalence of hypertension had non-significant difference in reproductive-aged women with PCOS [OR $(95 \% \mathrm{Cl}): 1.56(0.76,3.06), \mathrm{P}=0.198]$. Besides, there was no significant difference in BMI of $<25 \mathrm{~kg} / \mathrm{m}^{2}$ [OR $(95 \% \mathrm{Cl}): 1.33(0.12,15.14), \mathrm{P}=0.819$ ], $\mathrm{BMl}$ of $\geq 30 \mathrm{~kg} / \mathrm{m}^{2}$ [OR $(95 \% \mathrm{Cl}): 1.53$ $(0.90,2.61), P=0.116]$, and $\mathrm{BMI}$ of $25-<30 \mathrm{~kg} / \mathrm{m}^{2}$ [OR $(95 \% \mathrm{Cl}): 1.85(0.80,4.28), \mathrm{P}=0.153$ ].

\section{Lipid profiles}

Figure 5-8 are forest plots summarizing comparison of HDL-C, TG, nonHDL-C and LDL-C, respectively. As shown in Figure 5 and 6 , reproductive-aged women with PCOS had significantly difference on HDL-C [SMD (95\% Cl): $-0.24(-0.39,-0.09), \mathrm{P}=0.002]$ and TG [SMD $(95 \% \mathrm{Cl}): 0.37(0.26,0.48), \mathrm{P}<0.001]$. The subgroup analysis of TG, BMI of $<25 \mathrm{~kg} / \mathrm{m}^{2}$ [SMD (95\% Cl): $\left.0.34(0.20,0.49), \mathrm{P}<0.001\right]$, BMI of $\geq 30$ $\mathrm{kg} / \mathrm{m}^{2}$ [MD (95\% Cl): $\left.0.54(0.31,0.78), \mathrm{P}<0.001\right]$, and BMl of $25-<30 \mathrm{~kg} / \mathrm{m}^{2}$ [MD (95\% Cl): $0.25(0.06$, $0.43), P=0.008]$ were increased in reproductive-aged women with PCOS. Meanwhile, the subgroup analysis showed that HDL-C significantly increased in BMI of $<25 \mathrm{~kg} / \mathrm{m}^{2}$ [SMD ( $\left.95 \% \mathrm{Cl}\right):-0.30(-0.50$, $-0.11), \mathrm{P}=0.002]$, and BMI of $25-30 \mathrm{~kg} / \mathrm{m}^{2}$ [MD (95\% Cl): $\left.-0.33(-0.65,-0.01), \mathrm{P}=0.041\right]$. However, BMI of $\geq 30 \mathrm{~kg} / \mathrm{m}^{2}$ had no significant difference [MD $\left.(95 \% \mathrm{Cl}): 0.07(-0.26,0.40), \mathrm{P}=0.664\right]$.

Figure 7 and 8 are forest plots summarizing comparison of nonHDL-C and LDL-C, respectively. As shown in Figure 7 and 8, reproductive-aged women with PCOS had significant difference on nonHDL-C [SMD (95\% Cl): 0.38 (0.29, 0.47), P < 0.001] and LDL-C [SMD (95\% Cl): $0.25(0.17,0.34), P<0.001]$. The 
subgroup analysis showed that nonHDL-C increased in BMI of $<25 \mathrm{~kg} / \mathrm{m}^{2}$ [SMD (95\% Cl): $0.37(0.24$, $0.50), \mathrm{P}<0.001]$, BMI of $\geq 30 \mathrm{~kg} / \mathrm{m}^{2}$ [MD (95\% Cl): $\left.0.49(0.30,0.69), \mathrm{P}<0.001\right]$, and BMl of $25-<30 \mathrm{~kg} / \mathrm{m}^{2}$ [MD (95\% Cl): $0.33(0.24,0.50), \mathrm{P}<0.001]$. In addition, LDL-C increased in BMI of $<25 \mathrm{~kg} / \mathrm{m}^{2}$ [SMD $(95 \%$ $\mathrm{Cl}): 0.28(0.15,0.41), \mathrm{P}<0.001]$ and $\mathrm{BMI}$ of $\geq 30 \mathrm{~kg} / \mathrm{m}^{2}[\mathrm{MD}(95 \% \mathrm{Cl}): 0.25(0.06,0.44), \mathrm{P}=0.010]$. However, LDL-C did not increase in BMI of $25-330 \mathrm{~kg} / \mathrm{m}^{2}$ [MD (95\% Cl): $0.19(-0.01,0.39), \mathrm{P}=0.066$ ].

\section{Publication bias}

There was no publication bias for SBP (asymmetry test $P=0.510$ ), DBP (asymmetry test $P=0.092$ ), HDL (asymmetry test $P=0.405$ ), TG (asymmetry test $P=0.633$ ), nonHDL-C (asymmetry test $P=0.938$ ), and LDL (asymmetry test $P=0.628$ ) according to the results of Egger's regression test.

\section{Discussion}

The novelty of this systematic review from 34 observational studies reports the correlation between cardiovascular risk and BMI categories for reproductive-aged women with PCOS. To the best of our knowledge, it is the first time that BMI categories were evaluated cardiovascular risk factors in reproductive-aged women with PCOS. There are four main results in the present meta-analysis: (1) baseline blood pressure increased in reproductive-aged women with PCOS, but there was non-significant difference on hypertension prevalence; (2) increased LDL-C, low HDL-C, and increased TG + increased nonHDL-C were seen in reproductive-aged women with PCOS; (3) increased cardiovascular risk was seen in reproductive-aged women with PCOS independent of BMI categories; (4) DBP and hypertension prevalence had no difference in all BMI categories.

In present meta-analysis, increased baseline blood pressure was seen in reproductive PCOS women, but there was no significant difference on hypertension prevalence. There was no relationship between blood pressure and BMI categories in reproductive-aged women with PCOS.

The study of Zimmermann et al. reported that BMI failed to reveal an association between reproductiveaged women with PCOS and hypertension [53]. Hudecova et al. reported that after adjusting for BMI, reproductive-aged women with PCOS had higher daytime systolic blood pressure, mean arterial values of blood pressure, and an increased pulse rate irrespectively of clinically verified hypertension [54]. Moreover, a study from Australia reported that hypertension in reproductive PCOS women was not associated with $\mathrm{BMI}$, giving further information that the cardiometabolic abnormalities may be independent of weight in PCOS [32]. However, previous studies showed that the prevalence of hypertension increased in premenopausal [55] and perimenopausal PCOS women [56]. Schmidt et al. showed that hypertension and hypertriglyceridemia were the only cardiovascular risk factor that persisted more frequently in postmenopausal PCOS women. Therefore, hypertension is more prevalent in PCOS women from the fourth decade of life in comparison to respective controls [55]. 
There are some mainly factors for increased blood pressure in PCOS women, including endothelial dysfunction and decreased vascular compliance [57]. The increased risk of hypertension was explained by insulin resistance and hyperinsulinemia that exert hypertrophy of vascular muscle wall and reduce compliance by interference with endothelium-dependent vasodilatation mechanisms [58].

Hyperinsulinemia advocated endothelin 1 (ET-1) production, meanwhile, it also influences the hypertrophic effect on vascular endothelial and smooth muscle cells [57]. Besides, ET-1 is commonly chronically increased in women with PCOS, which regulates endothelial function.

In weight-matched studies, our results showed that increased LDL-C, low HDL-C, and increased TG + increased nonHDL-C were seen in reproductive-aged women with PCOS. Moreover, dyslipidemia in reproductive PCOS women had no relationship with body weight. The outcomes consistent with that of the previous studies $[9,59]$. PCOS women younger than 40 appears to have dyslipidemia [59]. Therefore, PCOS per se increases lipid levels, though the absolute value and the related cardiovascular risk may be different between individual patients. This is an important finding that lipid pattern assessment for decreased cardiovascular risk in reproductive PCOS women at all BMI categories.

The present meta-analysis reported that higher LDL-C was found in normal-weight (BMI of $<25 \mathrm{~kg} / \mathrm{m}^{2}$ ) and obese reproductive-aged women with PCOS (BMI of $\geq 30 \mathrm{~kg} / \mathrm{m}^{2}$ ), and low HDL-C was seen in normal-weight and overweight (BMI of $25-30 \mathrm{~kg} / \mathrm{m}^{2}$ ) subjects. Moreover, reproductive PCOS women at all BMI categories increased TG and nonHDL-C levels, including normal-weight, overweight and obesity. The subset analyses from a meta-analysis showed higher LDL-C was found in women with higher BMI categories [60]. However, our outcomes of reproductive PCOS women showed that increased LDL-C was seen in normal-weight and obese subjects. Meanwhile, nonHDL-C increased in all BMI categories.

Because the measurement of LDL-C can be influenced by increased TG in cardiovascular risk assessment [61], an appropriate way to estimate the amount of apoB-containing lipoprotein is the determination of nonHDL-C [62].

The level of nonHDL-C has a comparable prognostic relevance, similar to LDL-C $[63,64]$. It is necessary to detect the nonHDL-C level in reproductive PCOS women. Higher nonHDL-C levels were generally found in this population whose mean BMI was higher, but differences were also clearly found with PCOS even the $\mathrm{BMI}$ was in the normal-weight and overweight categories. The increasing level of nonHDL-C predict longterm cardiovascular risk, particularly at a young age. The suggests that obesity or overweight is not the absolute factor for reproductive PCOS women. Because, atherosclerotic process starts early life during life, this confirms the need to assess and eventually treat altered lipid profile in young women with PCOS [7].

Although obesity is an important confounder in relationship between PCOS and cardiovascular risk, nonobese subjects also have a high dyslipidemia risk $[57,65]$. In a meta-analysis of approximately 15000 women from 35 studies, women with PCOS had an increased prevalence of overweight, obesity and central obesity [57]. In addition to the reproductive and psychological characteristics of PCOS, the metabolic aberrations of PCOS worsen with obesity [66]. However, non-obese women with PCOS are also 
at an increased risk of similar cardiometabolic aberrations. Therefore, it is necessary to screened lipid levels for reproductive PCOS women with all BMI categories.

\section{Limitation}

Several limitations should also be noted. First, PCOS is a heterogeneous disease and the phenotypes are unable to exhibit in this meta-analysis. Second, there was a significant clinical and statistical heterogeneity in the pooled analysis. This could be due to confounding effects related to factors such as age, BMI, study quality, ethnicity, PCOS phenotypes and other clinical features. Besides, the use of country regions as a proxy for ethnicity due to lack of reporting of ethnic compositions in most studies. Further studies should include ethnicity of participants to allow for better understanding on the differential effects of this on PCOS or the metabolic syndrome. Third, the Egger's test has low power when there are less than 10 studies in the meta-analysis [67]. Our analyses included more than 10 studies for blood pressure and lipid profile, but less than ten studies for hypertension prevalence, which may have resulted in too low power to detect asymmetry.

\section{Conclusion}

In conclusion, our meta-analysis suggests that baseline blood pressure increases in reproductive-aged women with PCOS, but prevalence of hypertension has non-significantly difference. Besides, dyslipidemia is common in reproductive-aged women with PCOS and is characterized not only by low HDL-C and increased TG but also by high LDL-C and nonHDL-C. These lipid profiles are worse in reproductive-aged women with PCOS compared with controls, independent of BMI categories.

\section{Declarations}

\section{Consent for publication}

The present study was approved by the Ethics Committee Board of Lanzhou University Second Hospital (D2019-098).

\section{Competing interests}

No potential conflict of interest was reported by the authors.

\section{Funding}

This work was supported by the National Natural Science Foundation of China (81960086).

\section{Author Contributions}

Chenchen Zhuang and Jing Yu conceived the manuscript. Chenchen Zhuang designed the study and wrote the initial draft of manuscript. Chenchen Zhuang, Qiongying Wang, Wenjuan Wang and Runmin 
Sun analyzed the data. Qiongying Wang and Wenjuan Wang collected the data. All authors reviewed the manuscript.

\section{Acknowledgements}

We are grateful to Associate Ph.D. Jumo Zhu (University Medical Center of Groningen) and Ph.D. Ziyi Guo (University of New South Wales) for his assistance in the field. We also acknowledge Prof. Yuanhang Cheng (City University of Hong Kong) for constructive suggestions. Extremely careful and thoughtful reviews by Associate Editor and Reviewer improved this manuscript greatly.

\section{References}

1. Cooney LG, Dokras A. Beyond fertility: polycystic ovary syndrome and long-term health. Fertility and sterility. 2018;110(5):794-809.

2. Bozdag G, Mumusoglu S, Zengin D, Karabulut E, Yildiz BO. The prevalence and phenotypic features of polycystic ovary syndrome: a systematic review and meta-analysis. Human reproduction (Oxford, England). 2016;31(12):2841-55.

3. Obesity and reproduction: an educational bulletin. Fertility and sterility. 2008;90(5 Suppl):S21-9.

4. Behboudi-Gandevani S, Ramezani Tehrani F, Hosseinpanah F, Khalili D, Cheraghi L, Kazemijaliseh H, et al. Cardiometabolic risks in polycystic ovary syndrome: long-term population-based follow-up study. Fertility and sterility. 2018;110(7):1377-86.

5. Sirmans SM, Pate KA. Epidemiology, diagnosis, and management of polycystic ovary syndrome. Clinical epidemiology. 2013;6:1-13.

6. Pencina MJ, Navar AM, Wojdyla D, Sanchez RJ, Khan I, Elassal J, et al. Quantifying Importance of Major Risk Factors for Coronary Heart Disease. Circulation. 2019;139(13):1603-11.

7. Wild RA, Carmina E, Diamanti-Kandarakis E, Dokras A, Escobar-Morreale HF, Futterweit W, et al. Assessment of cardiovascular risk and prevention of cardiovascular disease in women with the polycystic ovary syndrome: a consensus statement by the Androgen Excess and Polycystic Ovary Syndrome (AE-PCOS) Society. The Journal of clinical endocrinology and metabolism. 2010;95(5):2038-49.

8. Shroff R, Kerchner A, Maifeld M, Van Beek EJ, Jagasia D, Dokras A. Young obese women with polycystic ovary syndrome have evidence of early coronary atherosclerosis. The Journal of clinical endocrinology and metabolism. 2007;92(12):4609-14.

9. Wild RA, Painter PC, Coulson PB, Carruth KB, Ranney GB. Lipoprotein lipid concentrations and cardiovascular risk in women with polycystic ovary syndrome. The Journal of clinical endocrinology and metabolism. 1985;61(5):946-51.

10. Carmina E. Cardiovascular risk and events in polycystic ovary syndrome. Climacteric : the journal of the International Menopause Society. 2009;12 Suppl 1:22-5. 
11. Moran LJ, Misso ML, Wild RA, Norman RJ. Impaired glucose tolerance, type 2 diabetes and metabolic syndrome in polycystic ovary syndrome: a systematic review and meta-analysis. Human reproduction update. 2010;16(4):347-63.

12. Dokras A, Bochner M, Hollinrake E, Markham S, Vanvoorhis B, Jagasia DH. Screening women with polycystic ovary syndrome for metabolic syndrome. Obstetrics and gynecology. 2005;106(1):131-7.

13. Revised 2003 consensus on diagnostic criteria and long-term health risks related to polycystic ovary syndrome. Fertility and sterility. 2004;81(1):19-25.

14. Glueck CJ, Goldenberg N. Characteristics of obesity in polycystic ovary syndrome: Etiology, treatment, and genetics. Metabolism: clinical and experimental. 2019;92:108-20.

15. Jeanes $Y M$, Reeves $S$. Metabolic consequences of obesity and insulin resistance in polycystic ovary syndrome: diagnostic and methodological challenges. Nutrition research reviews. 2017;30(1):97-105.

16. Lim SS, Davies MJ, Norman RJ, Moran LJ. Overweight, obesity and central obesity in women with polycystic ovary syndrome: a systematic review and meta-analysis. Human reproduction update. 2012;18(6):618-37.

17. Clinical guidelines on the identification, evaluation, and treatment of overweight and obesity in adults: executive summary. Expert Panel on the Identification, Evaluation, and Treatment of Overweight in Adults. The American journal of clinical nutrition. 1998;68(4):899-917.

18. Moher D, Liberati A, Tetzlaff J, Altman DG. Preferred reporting items for systematic reviews and meta-analyses: the PRISMA statement. PLoS medicine. 2009;6(7):e1000097.

19. Wells G, Shea B, O'Connell D, Peterson j, Welch V, Losos M, et al. The Newcastle-Ottawa Scale (NOS) for Assessing the Quality of Non-Randomized Studies in Meta-Analysis. \. 2000;】.

20. Adali E, Yildizhan R, Kurdoglu M, Bugdayci G, Kolusari A, Sahin HG. Increased plasma thrombinactivatable fibrinolysis inhibitor levels in young obese women with polycystic ovary syndrome. Fertility and sterility. 2010;94(2):666-72.

21. Akram T, Hasan S, Imran M, Karim A, Arslan M. Association of polycystic ovary syndrome with cardiovascular risk factors. Gynecological endocrinology : the official journal of the International Society of Gynecological Endocrinology. 2010;26(1):47-53.

22. Alexandraki K, Protogerou AD, Papaioannou TG, Piperi C, Mastorakos G, Lekakis J, et al. Early microvascular and macrovascular dysfunction is not accompanied by structural arterial injury in polycystic ovary syndrome. Hormones (Athens, Greece). 2006;5(2):126-36.

23. Arikan S, Akay H, Bahceci M, Tuzcu A, Gokalp D. The evaluation of endothelial function with flowmediated dilatation and carotid intima media thickness in young nonobese polycystic ovary syndrome patients; existence of insulin resistance alone may not represent an adequate condition for deterioration of endothelial function. Fertility and sterility. 2009;91(2):450-5.

24. Berneis K, Rizzo M, Lazzarini V, Fruzzetti F, Carmina E. Atherogenic lipoprotein phenotype and lowdensity lipoproteins size and subclasses in women with polycystic ovary syndrome. The Journal of clinical endocrinology and metabolism. 2007;92(1):186-9. 
25. Cascella T, Palomba S, Tauchmanovà L, Manguso F, Di Biase S, Labella D, et al. Serum aldosterone concentration and cardiovascular risk in women with polycystic ovarian syndrome. The Journal of clinical endocrinology and metabolism. 2006;91(11):4395-400.

26. Cetinkalp S, Karadeniz M, Erdogan M, Zengi A, Cetintas V, Tetik A, et al. Apolipoprotein E gene polymorphism and polycystic ovary syndrome patients in Western Anatolia, Turkey. Journal of assisted reproduction and genetics. 2009;26(1):1-6.

27. Cussons AJ, Watts GF, Stuckey BG. Dissociation of endothelial function and arterial stiffness in nonobese women with polycystic ovary syndrome (PCOS). Clinical endocrinology. 2009;71(6):80814.

28. Diamanti-Kandarakis E, Alexandraki K, Piperi C, Protogerou A, Katsikis I, Paterakis T, et al. Inflammatory and endothelial markers in women with polycystic ovary syndrome. European journal of clinical investigation. 2006;36(10):691-7.

29. El-Kannishy G, Kamal S, Mousa A, Saleh O, Badrawy AE, Farahaty RE, et al. Endothelial function in young women with polycystic ovary syndrome (PCOS): Implications of body mass index (BMI) and insulin resistance. Obesity research \& clinical practice. 2010;4(1):e1-e82.

30. Erdoğan M, Karadeniz M, Alper GE, Tamsel S, Uluer H, Cağlayan O, et al. Thrombin-activatable fibrinolysis inhibitor and cardiovascular risk factors in polycystic ovary syndrome. Experimental and clinical endocrinology \& diabetes : official journal, German Society of Endocrinology [and] German Diabetes Association. 2008;116(3):143-7.

31. Erdogan M, Karadeniz M, Berdeli A, Tamsel S, Yilmaz C. The relationship of the interleukin- $6-174$ G>C gene polymorphism with cardiovascular risk factors in Turkish polycystic ovary syndrome patients. International journal of immunogenetics. 2009;36(5):283-8.

32. Joham AE, Boyle JA, Zoungas S, Teede HJ. Hypertension in Reproductive-Aged Women With Polycystic Ovary Syndrome and Association With Obesity. American journal of hypertension. 2015;28(7):847-51.

33. Kargili A, Karakurt F, Kasapoglu B, Derbent A, Koca C, Selcoki Y. Association of polycystic ovary syndrome and a non-dipping blood pressure pattern in young women. Clinics (Sao Paulo, Brazil). 2010;65(5):475-9.

34. Ketel IJ, Stehouwer CD, Henry RM, Serné EH, Hompes P, Homburg R, et al. Greater arterial stiffness in polycystic ovary syndrome (PCOS) is an obesity-but not a PCOS-associated phenomenon. The Journal of clinical endocrinology and metabolism. 2010;95(10):4566-75.

35. Legro RS, Kunselman AR, Dunaif A. Prevalence and predictors of dyslipidemia in women with polycystic ovary syndrome. The American journal of medicine. 2001;111(8):607-13.

36. Liang SJ, Liou TH, Lin HW, Hsu CS, Tzeng CR, Hsu MI. Obesity is the predominant predictor of impaired glucose tolerance and metabolic disturbance in polycystic ovary syndrome. Acta obstetricia et gynecologica Scandinavica. 2012;91(10):1167-72.

37. Luque-Ramírez M, Mendieta-Azcona C, Alvarez-Blasco F, Escobar-Morreale HF. Androgen excess is associated with the increased carotid intima-media thickness observed in young women with 
polycystic ovary syndrome. Human reproduction (Oxford, England). 2007;22(12):3197-203.

38. Macut D, Panidis D, Glisić B, Spanos N, Petakov M, Bjekić J, et al. Lipid and lipoprotein profile in women with polycystic ovary syndrome. Canadian journal of physiology and pharmacology. 2008;86(4):199-204.

39. Meyer C, McGrath BP, Teede HJ. Overweight women with polycystic ovary syndrome have evidence of subclinical cardiovascular disease. The Journal of clinical endocrinology and metabolism. 2005;90(10):5711-6.

40. Moran LJ, Hutchison SK, Meyer C, Zoungas S, Teede HJ. A comprehensive assessment of endothelial function in overweight women with and without polycystic ovary syndrome. Clinical science (London, England : 1979). 2009;116(10):761-70.

41. Ni RM, Mo Y, Chen X, Zhong J, Liu W, Yang D. Low prevalence of the metabolic syndrome but high occurrence of various metabolic disorders in Chinese women with polycystic ovary syndrome. European journal of endocrinology. 2009;161(3):411-8.

42. Oral B, Mermi B, Dilek M, Alanoğlu G, Sütçü R. Thrombin activatable fibrinolysis inhibitor and other hemostatic parameters in patients with polycystic ovary syndrome. Gynecological endocrinology : the official journal of the International Society of Gynecological Endocrinology. 2009;25(2):110-6.

43. Orio F, Jr., Palomba S, Cascella T, De Simone B, Di Biase S, Russo T, et al. Early impairment of endothelial structure and function in young normal-weight women with polycystic ovary syndrome. The Journal of clinical endocrinology and metabolism. 2004;89(9):4588-93.

44. Philbois SV, Gastaldi AC, Facioli TP, Felix ACS, Reis RMD, Fares TH, et al. Women with Polycystic Ovarian Syndrome Exhibit Reduced Baroreflex Sensitivity That May Be Associated with Increased Body Fat. Arquivos brasileiros de cardiologia. 2019;112(4):424-9.

45. Rizzo M, Longo RA, Guastella E, Rini GB, Carmina E. Assessing cardiovascular risk in Mediterranean women with polycystic ovary syndrome. Journal of endocrinological investigation. 2011;34(6):422-6.

46. Sasaki A, Emi Y, Matsuda M, Sharula, Kamada Y, Chekir C, et al. Increased arterial stiffness in mildlyhypertensive women with polycystic ovary syndrome. The journal of obstetrics and gynaecology research. 2011;37(5):402-11.

47. Soares GM, Vieira CS, Martins WP, Franceschini SA, dos Reis RM, Silva de Sá MF, et al. Increased arterial stiffness in nonobese women with polycystic ovary syndrome (PCOS) without comorbidities: one more characteristic inherent to the syndrome? Clinical endocrinology. 2009;71(3):406-11.

48. Sterling L, Liu J, Okun N, Sakhuja A, Sierra S, Greenblatt E. Pregnancy outcomes in women with polycystic ovary syndrome undergoing in vitro fertilization. Fertility and sterility. 2016;105(3):7917.e2.

49. Tarkun I, Arslan BC, Cantürk Z, Türemen E, Sahin T, Duman C. Endothelial dysfunction in young women with polycystic ovary syndrome: relationship with insulin resistance and low-grade chronic inflammation. The Journal of clinical endocrinology and metabolism. 2004;89(11):5592-6.

50. Tíras MB, Yalcìn R, Noyan V, Maral I, Yìldìrim M, Dörtlemez O, et al. Alterations in cardiac flow parameters in patients with polycystic ovarian syndrome. Human reproduction (Oxford, England). 
1999;14(8):1949-52.

51. Vryonidou A, Papatheodorou A, Tavridou A, Terzi T, Loi V, Vatalas IA, et al. Association of hyperandrogenemic and metabolic phenotype with carotid intima-media thickness in young women with polycystic ovary syndrome. The Journal of clinical endocrinology and metabolism. 2005;90(5):2740-6.

52. Yildiz BO, Haznedaroğlu IC, Kirazli S, Bayraktar M. Global fibrinolytic capacity is decreased in polycystic ovary syndrome, suggesting a prothrombotic state. The Journal of clinical endocrinology and metabolism. 2002;87(8):3871-5.

53. Zimmermann S, Phillips RA, Dunaif A, Finegood DT, Wilkenfeld C, Ardeljan M, et al. Polycystic ovary syndrome: lack of hypertension despite profound insulin resistance. The Journal of clinical endocrinology and metabolism. 1992;75(2):508-13.

54. Hudecova M, Holte J, Olovsson M, Larsson A, Berne C, Sundstrom-Poromaa I. Prevalence of the metabolic syndrome in women with a previous diagnosis of polycystic ovary syndrome: long-term follow-up. Fertility and sterility. 2011;96(5):1271-4.

55. Holte J, Gennarelli G, Berne C, Bergh T, Lithell H. Elevated ambulatory day-time blood pressure in women with polycystic ovary syndrome: a sign of a pre-hypertensive state? Human reproduction (Oxford, England). 1996;11(1):23-8.

56. Dahlgren E, Johansson S, Lindstedt G, Knutsson F, Oden A, Janson PO, et al. Reprint of: Women with polycystic ovary syndrome wedge resected in 1956 to 1965: a long-term follow-up focusing on natural history and circulating hormones. Fertility and sterility. 2019;112(4 Suppl1):e162-e70.

57. Lim SS, Kakoly NS, Tan JWJ, Fitzgerald G, Bahri Khomami M, Joham AE, et al. Metabolic syndrome in polycystic ovary syndrome: a systematic review, meta-analysis and meta-regression. Obesity reviews : an official journal of the International Association for the Study of Obesity. 2019;20(2):33952.

58. Executive Summary of The Third Report of The National Cholesterol Education Program (NCEP) Expert Panel on Detection, Evaluation, And Treatment of High Blood Cholesterol In Adults (Adult Treatment Panel III). Jama. 2001;285(19):2486-97.

59. Talbott E, Clerici A, Berga SL, Kuller L, Guzick D, Detre K, et al. Adverse lipid and coronary heart disease risk profiles in young women with polycystic ovary syndrome: results of a case-control study. Journal of clinical epidemiology. 1998;51(5):415-22.

60. Wild RA, Rizzo M, Clifton S, Carmina E. Lipid levels in polycystic ovary syndrome: systematic review and meta-analysis. Fertility and sterility. 2011;95(3):1073-9.e1-11.

61. Martin SS, Blaha MJ, Elshazly MB, Brinton EA, Toth PP, McEvoy JW, et al. Friedewald-estimated versus directly measured low-density lipoprotein cholesterol and treatment implications. Journal of the American College of Cardiology. 2013;62(8):732-9.

62. Di Angelantonio E, Sarwar N, Perry P, Kaptoge S, Ray KK, Thompson A, et al. Major lipids, apolipoproteins, and risk of vascular disease. Jama. 2009;302(18):1993-2000. 
63. Palazón-Bru A, Carbayo-Herencia JA, Simarro-Rueda M, Artigao-Ródenas LM, Divisón-Garrote JA, Molina-Escribano F, et al. Comparison Between Non-High-Density Lipoprotein Cholesterol and LowDensity Lipoprotein Cholesterol to Estimate Cardiovascular Risk Using a Multivariate Model. The Journal of cardiovascular nursing. 2018;33(6):E17-e23.

64. Palazón-Bru A, Folgado-de la Rosa DM, Cortés-Castell E, López-Cascales MT, Gil-Guillén VF. Sample size calculation to externally validate scoring systems based on logistic regression models. PloS one. 2017;12(5):e0176726.

65. Ma X, Hayes E, Prizant H, Srivastava RK, Hammes SR, Sen A. Leptin-Induced CART (Cocaine- and Amphetamine-Regulated Transcript) Is a Novel Intraovarian Mediator of Obesity-Related Infertility in Females. Endocrinology. 2016;157(3):1248-57.

66. Liou TH, Yang JH, Hsieh CH, Lee CY, Hsu CS, Hsu MI. Clinical and biochemical presentations of polycystic ovary syndrome among obese and nonobese women. Fertility and sterility. 2009;92(6):1960-5.

67. Pustejovsky JE, Rodgers MA. Testing for funnel plot asymmetry of standardized mean differences. Research synthesis methods. 2019;10(1):57-71.

\section{Figures}
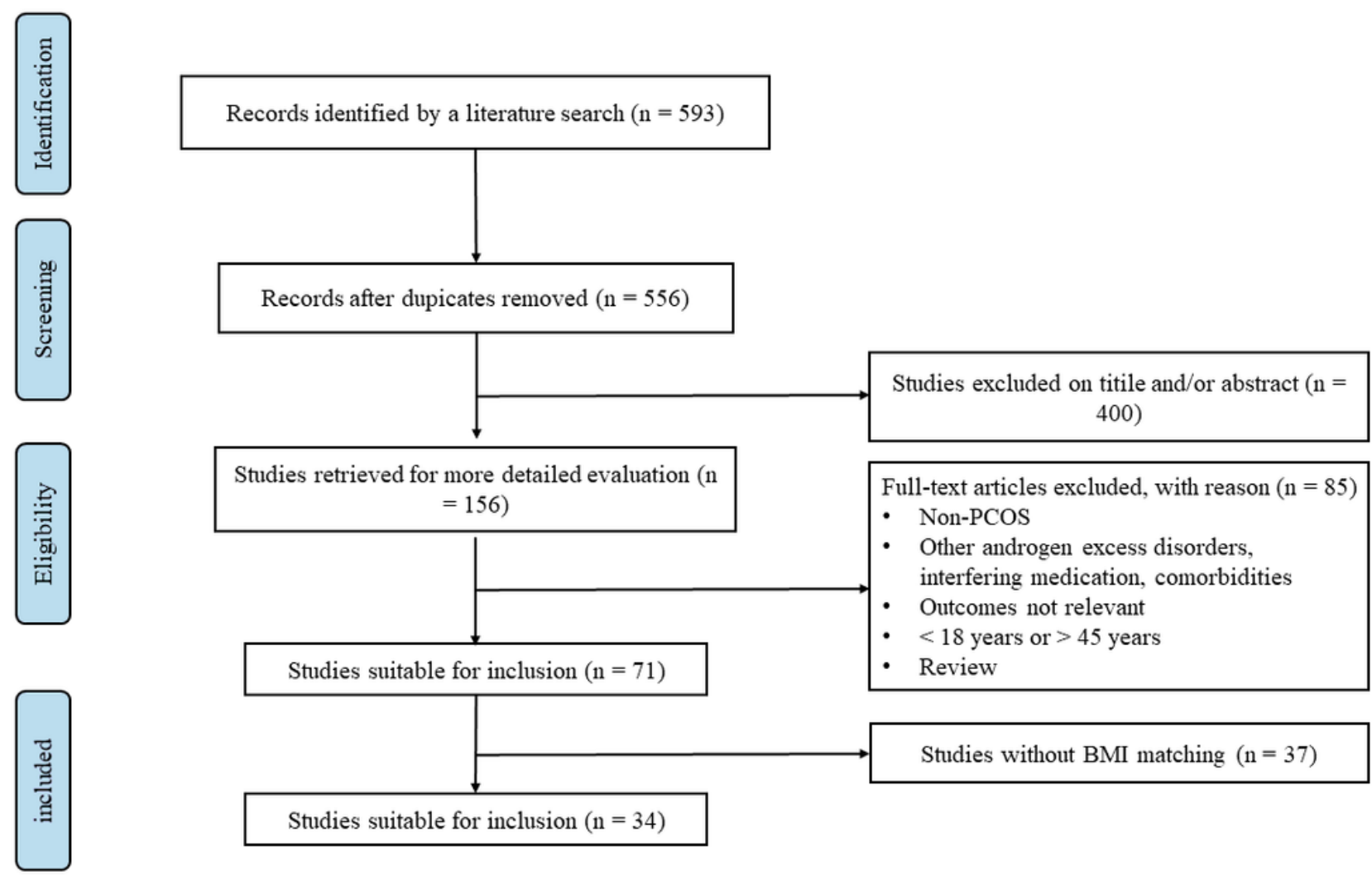

\section{Figure 1}

Flow chart of the study selection procedure. 
Study

ID

$\%$

WMD $(95 \% \mathrm{Cl}) \quad$ Weight

$\mathrm{BMI}<25$

Ni (2009)

Philbois (2019)

Sasaki (2011)

Soares (2009)

Adali (2010)

Cascella (2006)

Cussons (2009)

Legro (2001)

Subtotal $(I-$ squared $=43.0 \%, p=0.092)$

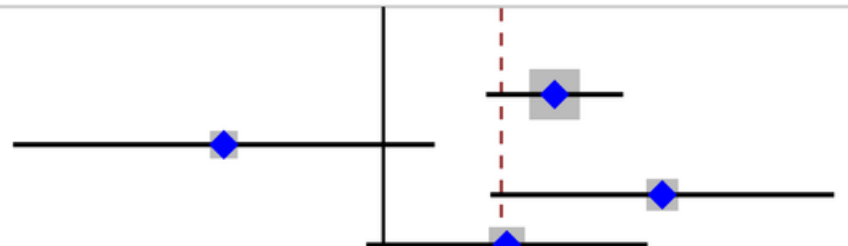

$4.30(2.58,6.02) \quad 21.83$

$-4.00(-9.29,1.29) 5.38$

$7.00(2.69,11.31) \quad 7.50$

$3.10(-0.43,6.63) \quad 10.11$

$4.48(-0.95,9.91) \quad 5.14$

$2.00(-2.19,6.19) \quad 7.86$

$2.00(-4.74,8.74) \quad 3.52$

$2.00(-3.02,7.02) \quad 5.87$

$3.02(1.11,4.94) \quad 67.21$

$\mathrm{BM} \mathrm{I} \geq 30$

Meyer (2005)

Legro (2001)

Subtotal (I-squared $=0.0 \%, p=0.503$ )

$25 \leq \mathrm{BMI}<30$

Alexandraki (2006)

Diamanti-Kandarakis (2006)

Vryonidou (2005)

Subtotal $(\mathrm{I}$-squared $=0.0 \%, p=0.775$ )

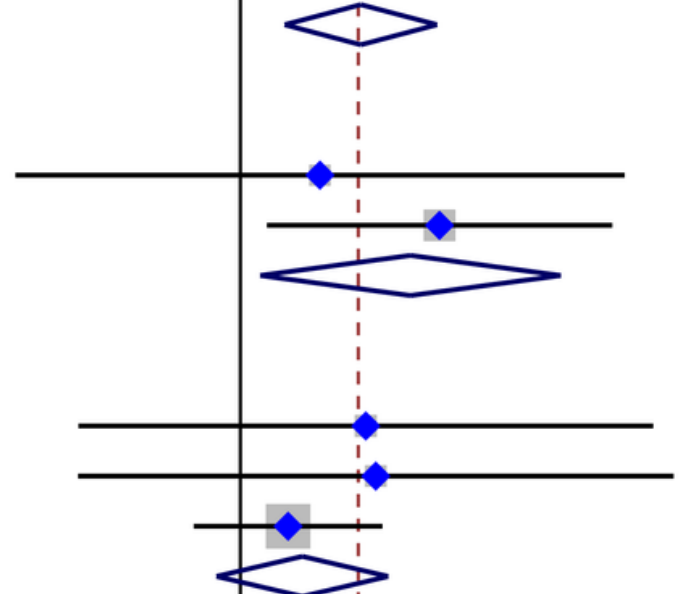

$2.00(-5.64,9.64) \quad 2.81$

$5.00(0.67,9.33) \quad 7.44$

$4.27(0.50,8.04) \quad 10.25$

Overall $(\mathrm{I}$-squared $=25.1 \%, p=0.190)$

NOTE: Weights are from random effects analysis

$-11.3$

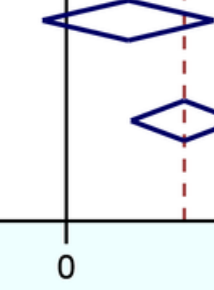

$3.15(-4.06,10.36) 3.12$

$3.40(-4.07,10.87) 2.93$

$1.20(-1.17,3.57) \quad 16.49$

$1.56(-0.60,3.71) \quad 22.54$

$2.96(1.62,4.30) \quad 100.00$

\section{Figure 2}

Forest plot showing the effect of systolic blood pressure in reproductive women with polycystic ovary syndrome (PCOS) ( $\mathrm{mmHg}$ ). Studies are classified by different body mass index (BMI) categories (BMI of $<25 \mathrm{~kg} / \mathrm{m} 2$, BMl of $\geq 30 \mathrm{~kg} / \mathrm{m} 2$ and BMl of $25-<30 \mathrm{~kg} / \mathrm{m} 2$ ). 
Study

ID

$\%$

WMD $(95 \% \mathrm{Cl}) \quad$ Weight

$\mathrm{BMI}<25$

Ni (2009)

Philbois (2019)

Sasaki (2011)

Soares (2009)

Adali (2010)

Cascella (2006)

Cussons (2009)

Legro (2001)

Subtotal $(\mathrm{I}$-squared $=72.2 \%, p=0.001$ )

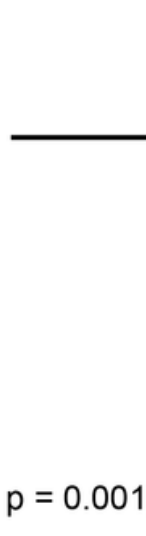

$\mathrm{BM} \mid \geq 30$

Meyer (2005)

Legro (2001)

Subtotal (I-squared $=0.0 \%, p=0.806$ )

$25 \leq \mathrm{BMI}<30$

Alexandraki (2006)

Diamanti-Kandarakis (2006)

Subtotal (I-squared $=0.0 \%, p=0.774$ )

Overall (I-squared $=62.1 \%, p=0.002)$

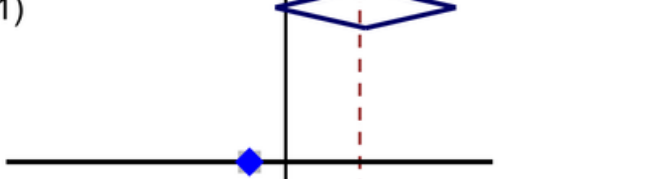

$4.70(3.36,6.04) \quad 14.50$

$-4.00(-9.04,1.04) 6.23$

$5.40(2.04,8.76) \quad 9.46$

$1.00(-1.96,3.96) \quad 10.42$

$-0.11(-4.17,3.95) 7.94$

$1.00(-0.75,2.75) \quad 13.54$

$2.00(-3.49,7.49) \quad 5.59$

$1.00(-3.52,5.52) \quad 7.08$

$1.76(-0.23,3.75) \quad 74.76$

$-0.80(-6.17,4.57) 5.76$

$0.00(-3.49,3.49) \quad 9.15$

$-0.24(-3.16,2.69) 14.91$

NOTE: Weights are from random effects analysis

$$
-9.95
$$

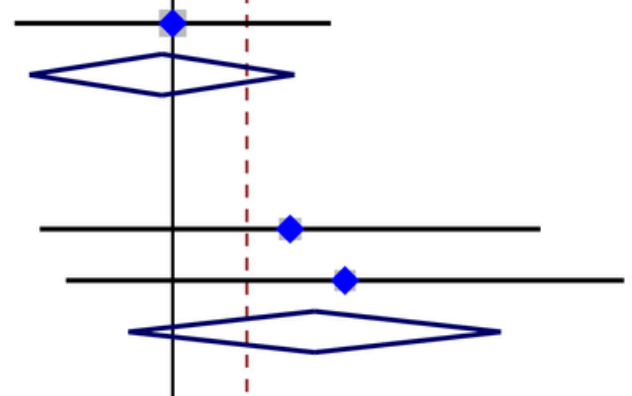

$2.59(-2.93,8.11) \quad 5.55$

$3.80(-2.35,9.95) \quad 4.79$

$3.13(-0.98,7.24) \quad 10.33$

$1.64(0.03,3.24) \quad 100.00$

\section{Figure 3}

Forest plot showing the effect of diastolic blood pressure in reproductive women with polycystic ovary syndrome (PCOS) ( $\mathrm{mmHg}$ ). Studies are classified by different body mass index (BMI) categories (BMI of $<25 \mathrm{~kg} / \mathrm{m} 2$, BMl of $\geq 30 \mathrm{~kg} / \mathrm{m} 2$ and BMl of $25-<30 \mathrm{~kg} / \mathrm{m} 2$ ). 


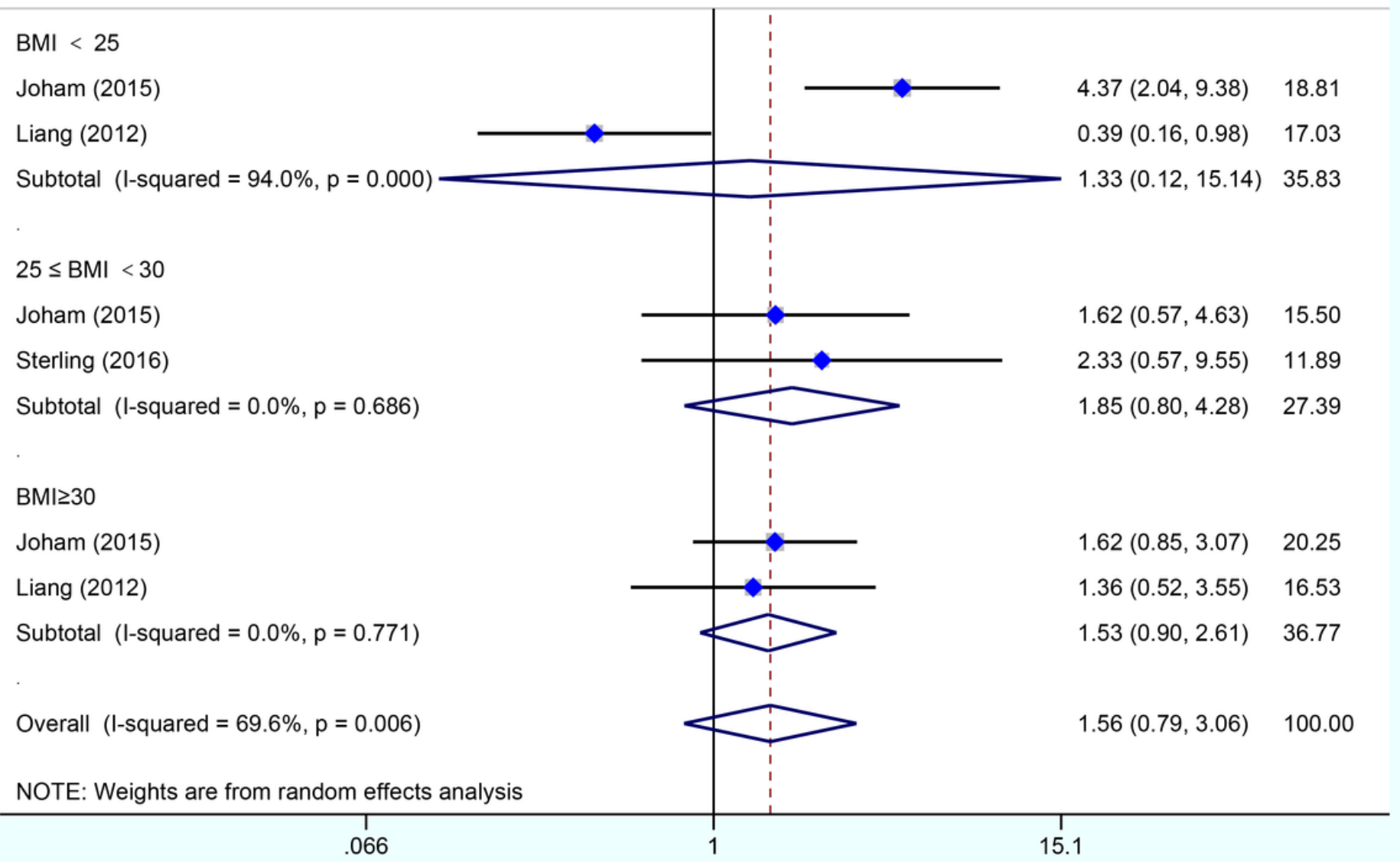

\section{Figure 4}

Forest plot of the association between reproductive polycystic ovary syndrome (PCOS) and hypertension prevalence. Studies are classified by different body mass index (BMI) categories (BMl of $<25 \mathrm{~kg} / \mathrm{m} 2, \mathrm{BMI}$ of $\geq 30 \mathrm{~kg} / \mathrm{m} 2$ and BMl of $25-<30 \mathrm{~kg} / \mathrm{m} 2$ ). 
Study

ID

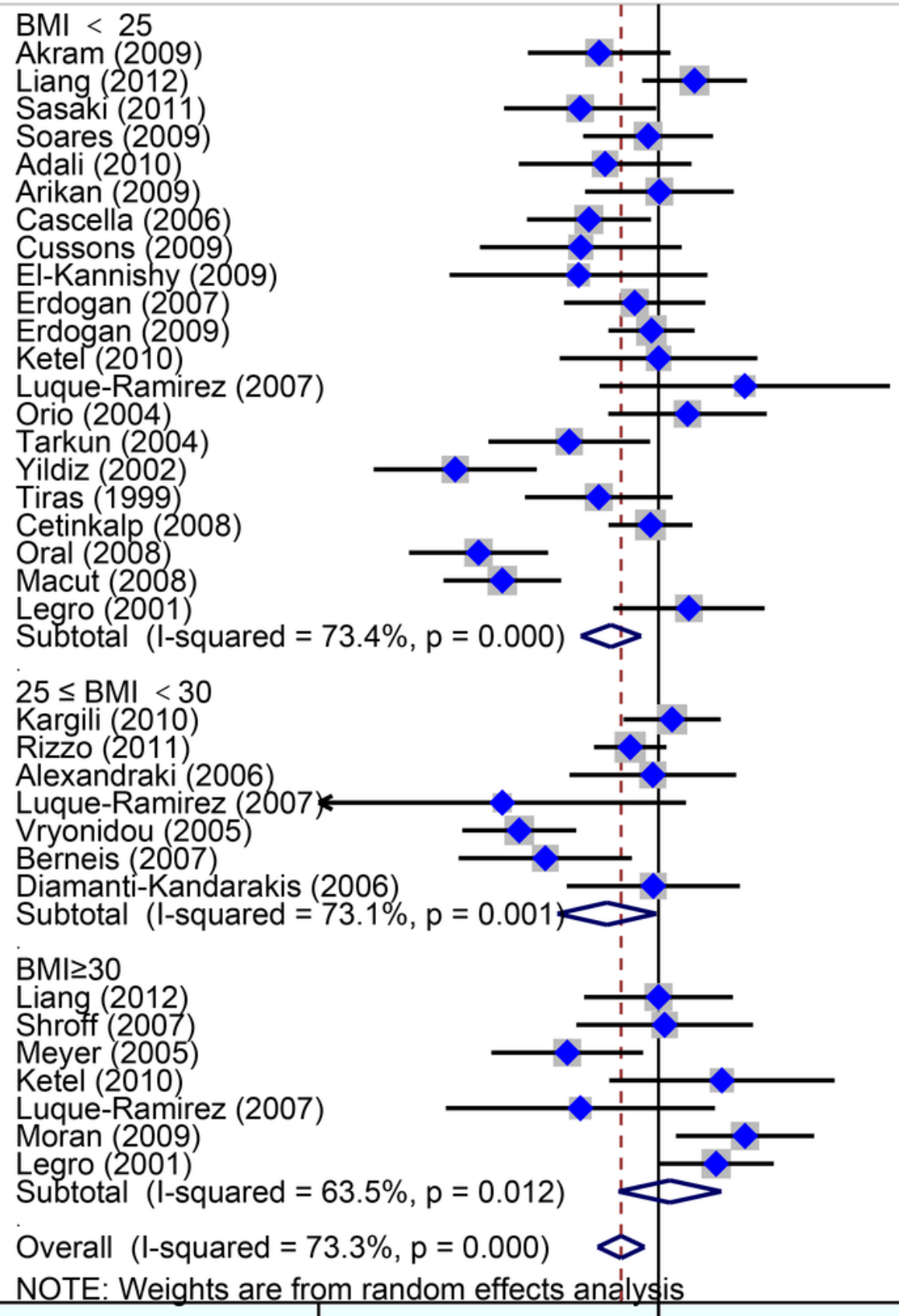

$\mathrm{BMI}<25$

Akram (2009)

Liang (2012)

Sasaki (2011)

Soares (2009)

Adali (2010)

Arikan (2009)

Cascella (2006)

Cussons (2009)

El-Kannishy (2009)

Erdogan (2007)

Erdogan (2009)

Ketel (2010)

Luque-Ramirez (2007)

Orio (2004)

Tarkun (2004)

Cetinkalp (2008)

Oral (2008)

Macut (2008)

Legro (2001)

$25 \leq \mathrm{BMI}<30$

Kargili (2010)

Rizzo (2011)

Alexandraki (2006)

Luque-Ramirez (2007)

ryonidou (2005)

Diamanti-Kandarakis $(2006)$

-

$\mathrm{BM} \mid \geq 30$

Ketel (2010)

Luque-Ramirez (2007)

Moran (2009)

Legro (2001)

Subtotal ( $\mathrm{I}$-squared $=63.5 \%, \mathrm{p}=0.012$ )

Overall (I-squared $=73.3 \%, p=0.000$ )

NOTE: Weights are from random effects analysis
SMD $(95 \% \mathrm{Cl}) \quad \stackrel{\%}{\text { Weight }}$

$-0.38(-0.84,0.08) 3.04$

$0.23(-0.10,0.57) 3.49$

$-0.50(-0.99,-0.01) 2.92$

$-0.07(-0.48,0.35) 3.19$

$-0.34(-0.90,0.21) 2.68$

$0.01(-0.47,0.48) 2.96$

$-0.45(-0.84,-0.05) 3.26$

$-0.50(-1.14,0.15) 2.37$

$-0.51(-1.34,0.31) 1.85$

$-0.15(-0.61,0.30) 3.05$

$-0.04(-0.32,0.23) 3.70$

$0.00(-0.63,0.63) 2.41$

$0.55(-0.38,1.48) \quad 1.61$

$0.19(-0.32,0.69) \quad 2.85$

$-0.57(-1.09,-0.05) 2.81$

$-1.30(-1.82,-0.78) 2.79$

$-0.38(-0.86,0.09) 2.98$

$-0.05(-0.32,0.22) 3.72$

$-1.15(-1.60,-0.71) 3.08$

$-1.00(-1.38,-0.62) 3.34$

$0.19(-0.29,0.68) 2.93$

$-0.30(-0.50,-0.11) 61.04$

$0.09(-0.22,0.40) \quad 3.58$

$-0.18(-0.41,0.05) 3.84$

$-0.04(-0.57,0.50) 2.75$

$-1.00(-2.18,0.18) 1.17$

$-0.89(-1.26,-0.53) 3.38$

$-0.72(-1.28,-0.17) 2.68$

$-0.03(-0.59,0.52) 2.68$

$-0.33(-0.65,-0.01) 20.08$

$0.00(-0.48,0.48) \quad 2.96$

$0.04(-0.53,0.61) \quad 2.64$

$-0.58(-1.07,-0.10) 2.93$

$0.41(-0.32,1.13) 2.14$

$-0.50(-1.36,0.36) 1.76$

$0.55(0.11,1.00) \quad 3.09$

$0.37(-0.00,0.74) \quad 3.37$

$0.07(-0.26,0.40) \quad 18.88$

$-0.24(-0.39,-0.09) 100.00$

\section{Figure 5}

Forest plot showing the effect of high-density lipoprotein-cholesterol (HDL-C) in reproductive women with polycystic ovary syndrome (PCOS). Studies are classified by different body mass index (BMI) categories (BMl of $<25 \mathrm{~kg} / \mathrm{m} 2$, BMl of $\geq 30 \mathrm{~kg} / \mathrm{m} 2$ and $\mathrm{BMl}$ of $25-<30 \mathrm{~kg} / \mathrm{m} 2$ ). 
Study

$\operatorname{SMD}(95 \% \mathrm{Cl}) \quad \stackrel{\%}{\text { Weight }}$

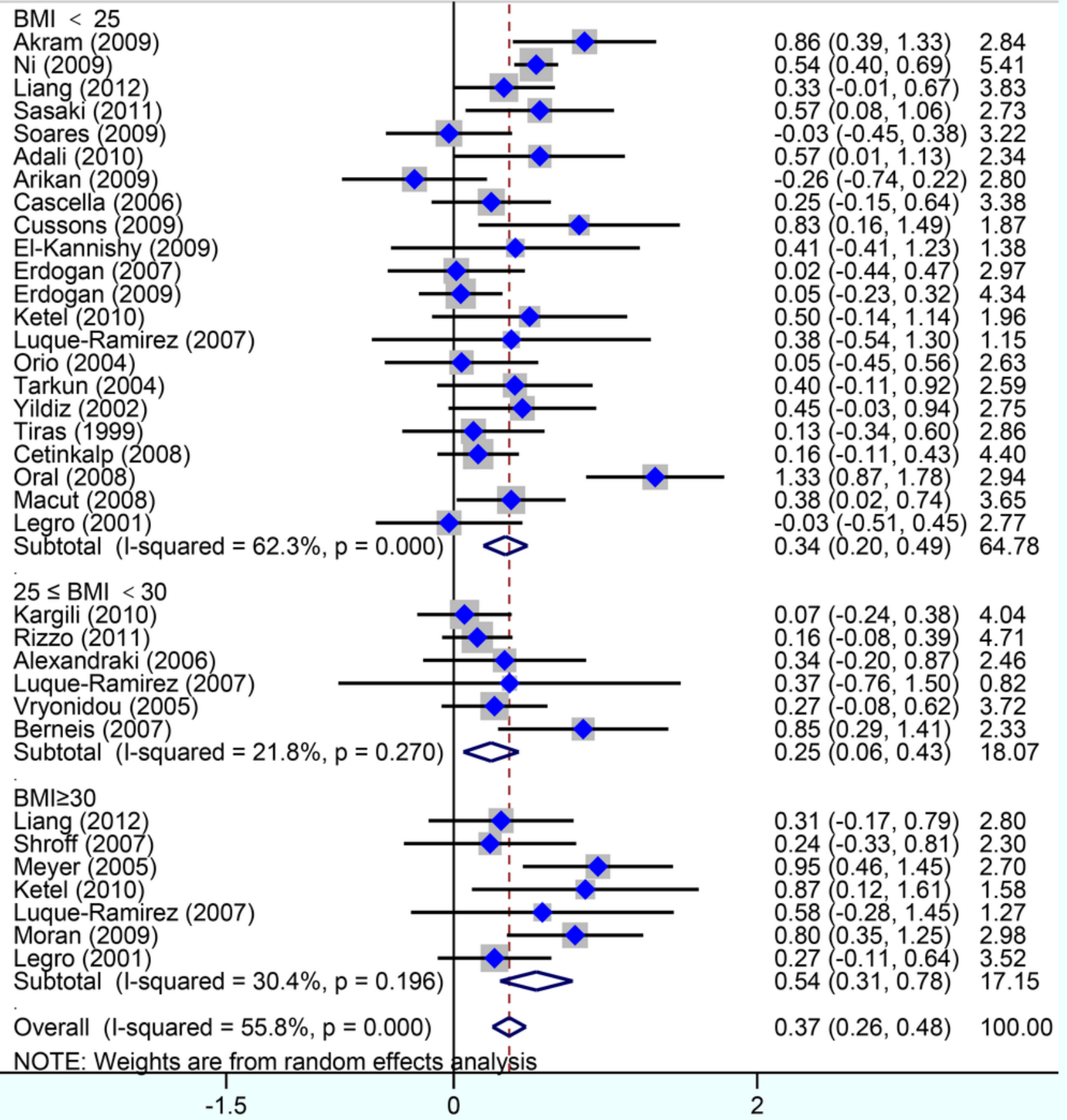

Figure 6

Forest plot showing the effect of triglyceride in reproductive women with polycystic ovary syndrome (PCOS). Studies are classified by different body mass index (BMI) categories (BMI of $<25 \mathrm{~kg} / \mathrm{m} 2$, BMI of $\geq 30 \mathrm{~kg} / \mathrm{m} 2$ and $\mathrm{BMI}$ of $25-<30 \mathrm{~kg} / \mathrm{m} 2)$. 
Study

$\operatorname{SMD}(95 \% \mathrm{Cl}) \quad \stackrel{\%}{\text { Weight }}$

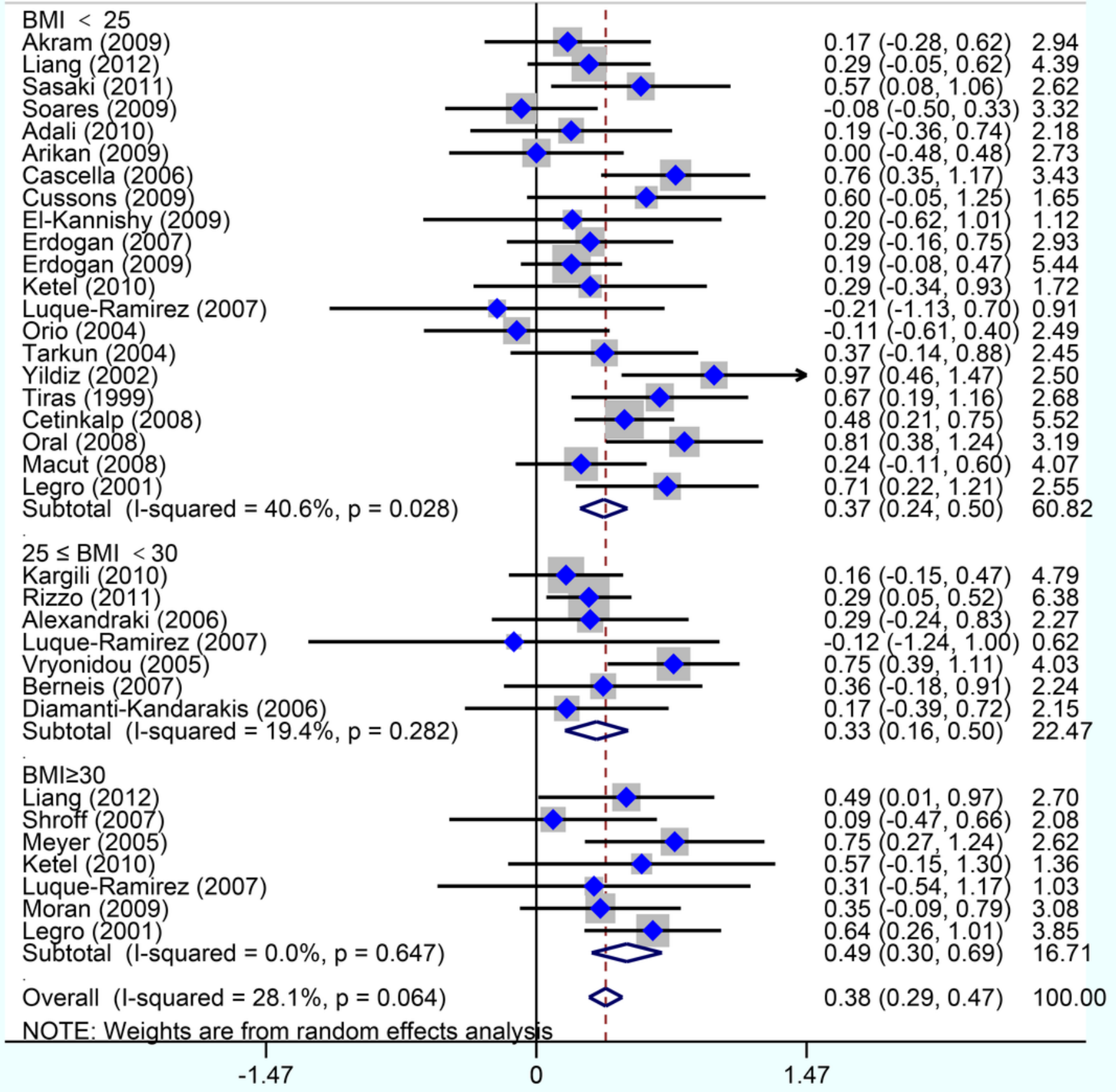

Figure 7

Forest plot showing the effect of nonhigh-density lipoprotein-cholesterol (nonHDL-C) in reproductive women with polycystic ovary syndrome (PCOS). Studies are classified by different body mass index (BMI) categories (BMl of $<25 \mathrm{~kg} / \mathrm{m} 2$, BMl of $\geq 30 \mathrm{~kg} / \mathrm{m} 2$ and BMl of $25-30 \mathrm{~kg} / \mathrm{m} 2$ ). 


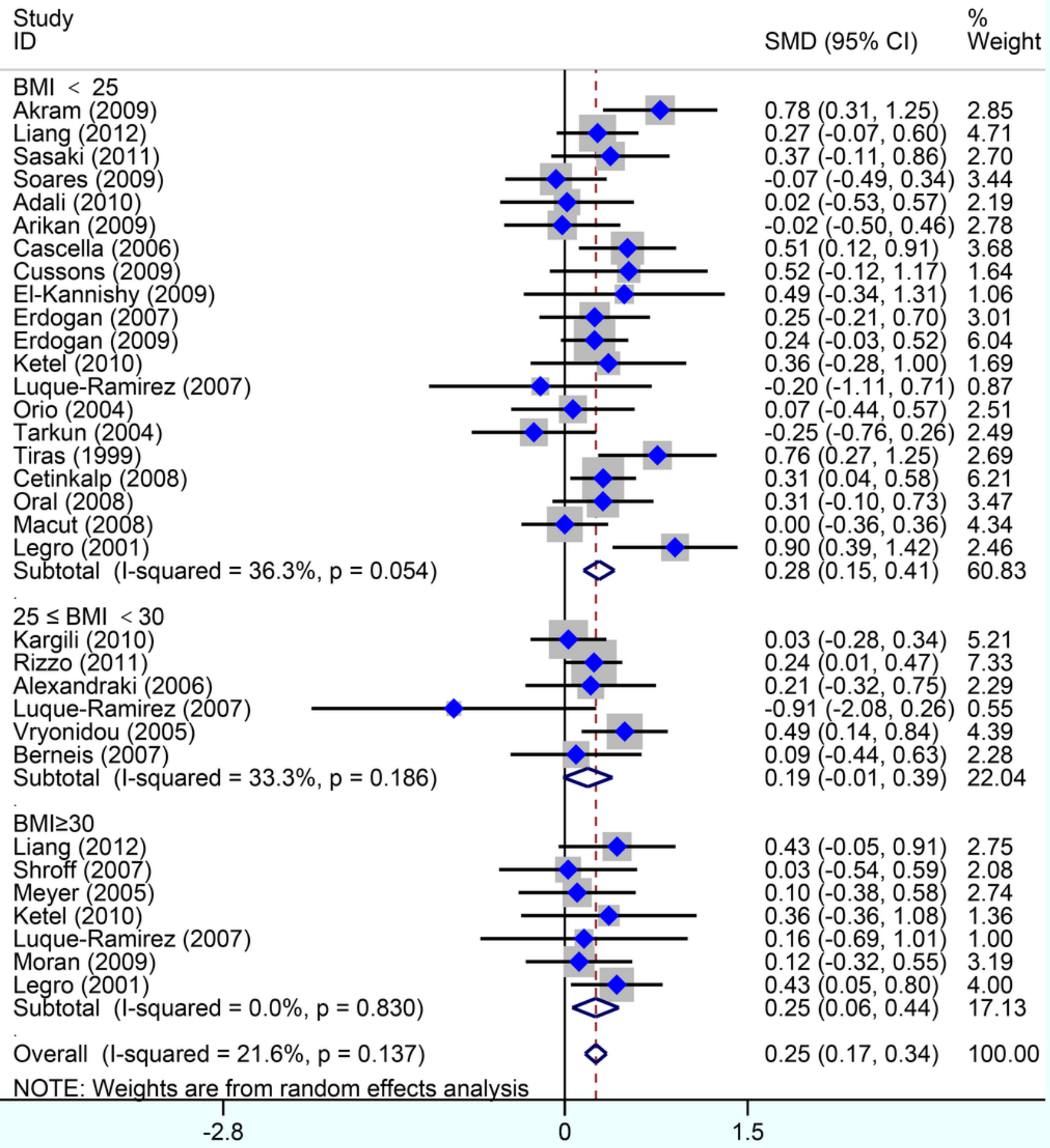

\section{Figure 8}

Forest plot showing the effect of low-density lipoprotein-cholesterol (LDL-C) in reproductive women with polycystic ovary syndrome (PCOS). Studies are classified by different body mass index (BMI) categories (BMl of $<25 \mathrm{~kg} / \mathrm{m} 2$, BMl of $\geq 30 \mathrm{~kg} / \mathrm{m} 2$ and BMl of $25-<30 \mathrm{~kg} / \mathrm{m} 2$ ). 